\title{
AVIFAUNA Y POTENCIAL PARA EL AVITURISMO DE LA CUENCA DEL MISHQUIYAQUILLO (REGIÓN SAN MARTÍN, AM AZONÍA PERUANA)
}

\author{
José Oriel ALTAMIRANO GUERRERO', Noam SHANY², José ÁLVAREZALONSO ${ }^{3}$
}

1 Asociación Misquiyaquillo, M oyobamba

2 Naturaleza y Cultura Internacional - $\mathrm{NCl}$

3 Instituto de Investigaciones de la Amazonía Peruana - IIAP, email: jalvarez@ iiap.org.pe

\section{RESUMEN}

Se presenta los resultados de la evaluación de la avifauna de la cuenca del Misquiyaquillo, afluente del río Mayo, Provincia de Moyobamba, Región San Martín, Perú. Esta zona, actualmente dentro de las áreas de conservación ambiental Almendra y Mishquiyacu-Rumiyacu, es una de las 128 IBAs (áreas importantes para las conservación de las aves) del Perú, y alberga un alto número de especies de gran interés para la conservación y para el aviturismo. La cuenca protege una muestra del típico bosque amazónico premontano, con altitudes que varían entre 900 y 1620 msnm, incluyendo bosques sobre arena blanca, bosque de cumbres con suelos pobres, y exóticos pastizales nativos. Se ha registrado en total 311 especies de aves, de las cuales 4 son endémicas para el Perú y 1 para el alto río Mayo, 15 son de distribución restringida, y un número considerable de especies raras y de distribución restringida. Por su accesibilidad por carretera (está a menos de $10 \mathrm{~km}$. de Moyobamba, capital de la Región San Martín) y la presencia de especies únicas esta área tiene un gran potencial para el aviturismo y el ecoturismo en general.

PALABRAS CLAVE: Avifauna, Misquiyaquillo Moyobamba, aves endémicas, aviturismo

\section{AVIFAUNA AND BIRDWATCHING POTENTIAL OF MISQUIYAQUILLO BASIN (SAN MARTIN REGION, PERUVIAN AMAZON)}

\begin{abstract}
We present here the results of the a study of the "Avifauna of Mishquiyaquillo basin, branch of Mayo River, Moyobamba Province, San Martin Region, Peru.” This area is within the Environmental Conservation Areas Almendra and Misquiyacu-Rumiyacu, and is one of the 128 IBAs (important bird areas) of Peru. The area harbors a high number of important species for conservation and avitourism. The habitat consists of a complex of typical lower montane forests ranging from 900 to $1620 \mathrm{~m}$, including white-sand forest and poor-soil ridge forest as well as native and exotic grasslands. 311 bird species have been recorded, 4 of them are endemic to Peru, and one endemic to upper Mayo River, 15 have restricted distribution in Peru and many more are rare or seldom encountered. Due to its proximity by a paved road to Moyobamba, the regional capital (less than $10 \mathrm{~km}$ from town center) and the diversity of bird life the area presents a unique potential for bird tourism and ecotourism.
\end{abstract}

KEYW ORDS: Avifauna, Misquiyaquillo Moyobamba, endemic birds, birdwatching 


\section{INTRODUCCIÓN}

La diversidad de aves en los cinco países que forman parte de los Andes Tropicales (Perú, Colombia, Ecuador, Venezuela y Bolivia) que comprenden apenas el $3 \%$ de la superficie mundial, alcanza cerca de 2780 especies, lo que corresponde al $85 \%$ de la diversidad continental de Sudamérica, y al $28 \%$ del total mundial (BirdLife, 2005); de esta cantidad de especies de aves, más de 1800 especies pueden ser vistas en el Perú (Clements \& Shany, 2001; Schulenberg et al., 2007; Plenge, 2011) y de éstas, más de 900 especies pueden ser vistas en el Alto Mayo (Plenge \& Williams, 2004).

Este importante número de especies hace que el Alto Mayo forme parte de la Ruta de Aves del Norte del Perú, con especies endémicas globalmente amenazadas como Xenoglaux loweryi, y Herpsilochmus parkeri, entre otras (PROMPERU, 2005).

En los últimos años, ante las crecientes amenazas para los bosques y la fauna silvestre por ampliación no planificada de la frontera agrícola ha impulsado una serie de iniciativas locales para la creación de espacios locales destinados a la conservación, como es el caso de las Áreas de Conservación Municipal, liderada en el Alto Mayo por la provincia de Moyobamba con 15 Áreas de Conservación Municipal (MPM 2004); en teoría, estas áreas garantizan la conservación y protección de importantes comunidades de aves, junto a otras especies de interés biológico y turístico, y la conservación de fuentes y nacientes de agua. Sin embargo, la mayoría de ellas no cuentan con ningún estudio previo que sustente biológicamente la creación ni ponga de relieve las potencialidades para el turismo, de forma que se promueva la sostenibilidad económica y su aprovechamiento sostenible compatible con los objetivos de conservación. Esta situación ha debilitado políticamente a estas áreas, muchas de las cuales sufren grandes presiones de colonos que talan ilegalmente el bosque, extracción ilegal de madera y de aprovechamiento insostenible de otros recursos.

En este contexto, evaluar y documentar la avifauna y otros atractivos ecoturísticos constituye una estrategia fundamental para promover la sostenibilidad financiera y la viabilidad de gestión de estas áreas de conservación. Esto incluye el desarrollo de actividades sostenibles dentro del área de conservación, especialmente el ecoturismo, la educación ambiental, la provisión y venta de servicios ambientales, y otras (Bolívar-Troncoso, 1999).

El 5 de abril del 2004 fueron creadas por la Municipalidad Provincial de Moyobamba -mediante sendas ordenanzas municipales- 15 áreas de conservación municipal - ACM en la de la provincia de Moyobamba. Entre ellas están el ACM Almendra, creada con O. M. N ${ }^{\circ}$ 065-MPM, y una extensión de 1620.95 ha, y el ACM Mishquiyacu-Rumiyacu, con O. M.No 071-MPM, y una extensión de 864.86 ha.

Esta iniciativa de creación de áreas de conservación municipal (hoy llamadas áreas de conservación ambiental) se complementa con la declaración de áreas importantes para la conservación de las aves en los Andes tropicales, conocidas por sus siglas en inglés como IBAs; se cuenta con 128 IBAs para el Perú, de las cuales 6 se encuentran en el departamento de San Martín, incluyendo una compartida con el departamento de Loreto y dos con Amazonas. Entre ellas está la IBA de Moyobamba, descrita como una zona que presenta un conjunto de hábitats, incluyendo bosque perennifolio tropical bajo, bosque de galería, bosque de arena blanca, bosque de palmas, cerrado, campos y pastizales estacionalmente inundados. En dicha IBA se propone la conservación y protección de 11 especies de aves, que incluyen especies globalmente amenazadas y endémicas, como Aburria aburri, Ara militaris, Touit stictoptera, Phaethornis koepckeae, Campylopterus villaviscensio, Heliodoxa gularis, Heliangelus regalis, Synallaxis cherriei, Hemitriccus rufigularis, Zimmerius villarejoi y Henicorhina leucoptera. Se menciona como una IBA desprotegida, con problemas de conservación y con amenazas como la pérdida de hábitat por la tala de áreas boscosas para la agricultura (Birdlife, 2005).

De las otras 5 áreas importantes para la conservación de las aves destacan dos más en el Alto Mayo: la IBA Jesús del Monte, ubicada entre el caserío Jesús del Monte y una parte del territorio del departamento de Loreto, con especies similares a la IBA Moyobamba, y la IBA Alto Mayo, ubicada en territorio del Bosque de Protección Alto Mayo (BirdLife, 2005).

Un importante trabajo de conservación y monitoreo tiene lugar en una cuenca que es parte del IBA Moyobamba, específicamente en quebrada Misquiyaquillo. Este trabajo es realizado por Asociación Ecosistemas Andinos (ECOAN), como parte del proyecto de monitoreo de aves Migratorias y Residentes en el Norte Peruano desarrollado entre los añas setiembre 2006 hasta Junio 2008 (datos no publicados).

\section{MATERIALY MÉTODOS}

\section{ÁREA DEESTUDIO}

La micro cuenca Mishquiyaquillose encuentra dividida por el divortium aquarum de las microcuencas del Rumiyacuy Almendra. Gran parte 
(la cuenca alta) se localiza dentro del Área de Conservación Municipal "Almendra"(creada por Ordenanza Municipal 065-MPM 13/02/04), mientras que la parte baja forma parte de distintas propiedades de particulares. Se ubica a la margen derecha del río Mayo, a $5 \mathrm{Km}$. de la ciudad de Moyobamba, siguiendo la carretera hacia Jepelacio, en el Sector Baños Termales de San Mateo.

La cuenca del Misquiyaquillo se encuentra entre los 900 a $1620 \mathrm{msnm}$, y cuenta con una variedad de pisos ecológicos y diferentes formaciones vegetales. En la zona se puede encontrar una gran variedad de hábitats, desde pasturas, plantaciones de café y bosque secundario en diversos estadios de recuperación, hasta varios tipos de bosques primarios, que incluyen bosques típicos premontanos y otras formaciones vegetales como bosques raquíticos sobre suelos pobres en cumbres de colinas, y bosques sobre arena blanca (incluyendo vegetación arbustiva y pastizales naturales o 'pajonales', ecosistemas sumamente raros en la Amazonía peruana, y especialmente en el pie de monte andino). Las formaciones boscosas ocupan una extensión aproximada de unas 300 ha. La quebrada de Misquiyaquillo desemboca en la parte media de la quebrada, la que es afluente de río Mayo.

La principal actividad económica en la microcuenca del Misquiyaquillo son los cultivos, la mayor parte de café; 21 familias campesinas trabajan en este lugar, con un promedio de dos hectáreas de café por familia, además de algunas extensiones pequeñas dedicadas a cultivos de pan llevar. La parte alta de la microcuenca es la más intervenida para fines agrícolas.

Respecto al clima, la temperatura promedio mensual varía entre $27.2^{\circ} \mathrm{C}$ y $29.0^{\circ} \mathrm{C}$, la precipitación anual promedio anual varía entre 1600 y 2000 mm (1354 $\mathrm{mm}$ en promedio en la estación meteorológica de Moyobamba). La humedad relativa (promedio mensual) está entre $81 \%$ en los meses de julio a noviembre y $86 \%$ en el mes de febrero. La zona de vida corresponde a bosque húmedo pre montano tropical (bh-PT; Holdridge, 1967).

La microcuenca del Misquiyaquillo se encuentra localizada en la Zona de Depresión Mayo - Huallaga, que data del Mesozoico (ONERN, 1986). La litología está representada por arcillas y arenisca arcillosas, areniscas cuarzosas y calizas, las mismas que pueden ser apreciadas en afloramientos sobre el camino que conduce a las partes altas de la cuenca.

La topografía de la zona es ondulada, dominada por colinas empinadas y pequeñas terrazas. Se distinguen suelos aluviales antiguos (terrazas bajas de pequeñas extensiones), especialmente en la parte baja de la cuenca. Son suelos que varían de rojizos a pardo rojizos oscuros y muy oscuros. La textura varía desde franco arenosos hasta franco arcillosos o arcilla. Las pendientes varían de 15 a 75\%. La reacción del suelo es fuertemente ácida (MPM, 2002).

\section{MATERIAL}

Se usó binoculares para las detecciones visuales y una grabadora (Sony TCD 5000) con micrófono direccional (Sennheiser ME88) para la grabación de cantos, que fueron comparados posteriormente. Las grabaciones de cantos más resaltantes serán depositadas en la Biblioteca de Sonidos Naturales, Laboratorio de Ornitología de Cornell, (Ithaca, NY) y las fotos en VIREO (Philadelphia, PA).

\section{MÉTODOS}

La metodología de evaluación fue la usada habitualmente en evaluaciones ornitológicas rápidas en bosques tropicales: recorridos a paso lento por las trochas desde primeras horas de la mañana hasta primeras horas de la tarde, durante los cuales se registró toda observación visual y auditiva de las especies presentes. Los registros incluyeron los siguientes datos: especie, tipo de observación (auditiva, visual), hora de observación, distancia aproximada al observador (distancia perpendicular a la trocha), número de individuos, tipo de hábitat, posición en el estrato de vegetación, y comportamiento (en el caso de que fuese de notar). Las evaluaciones comenzaban con las primeras luces del Amanecer (entre 5.15 y $5.30 \mathrm{am}$ ) y terminaban a las 2 ó 3 de la tarde, dependiendo de lo activas que estuviesen las aves.

Se usó ocasionalmente algunas redes de neblina en hábitats de particular interés, para ayudar en la identificación de algunas especies de sotobosque. Algunas especies fueron también fotografiadas.

Para las evaluaciones se usó dos técnicas básicas: conteo de puntos y transectos.

\section{CONTEO DE PUNTOS}

El método de conteo de puntos (Ralph \& Scout, 1981; Ralph et al., 1993) se basa en hacer paradas por períodos fijos de tiempo en lugares seleccionados al azar o en un diseño sistemático dentro de los hábitats de interés para registrar la presencia y abundancia de especies de aves, usualmente a cierta distancia del punto. En cada punto, también se registra el tipo de hábitat. Para asegurar que las especies registradas están realmente en un hábitat específico, por ejemplo café bajo sombra versus bosque sobre otro tipo de suelo, se anotó solamente los pájaros registrados visualmente u oídos hasta una distancia de $50 \mathrm{~m}$. del punto. 
En el presente estudio se contó las especies por diez minutos en cada parada, que estuvo separada de otras por al menos $250 \mathrm{~m}$ para reducir la posibilidad de contar el mismo individuo dos veces, y para hacer los conteos de punto independientes estadísticamente. Descartamos del análisis los individuos potencialmente duplicados, por ejemplo, aquéllos que se estaban moviendo en la dirección del siguiente punto de conteo para especies registradas en el siguiente punto.

\section{TRANSECTOS}

Esta técnica es usada ampliamente en las evaluaciones de fauna silvestre. Los transectos fueron trazados al azar en el área de estudio. Los transectos fueron de unos $1,500 \mathrm{~m}$. de longitud; esto corresponde aproximadamente a la longitud de los parches de pasturas naturales. Cuando fue posible, y para evitar mayor impacto en el bosque y en la avifauna, los transectos fueron trazados a lo largo de trochas ya existentes y fueron evaluados una vez al día. Las evaluaciones fueron realizadas entre las 5:30 y las 11 de la mañana, lo que corresponde con las horas de más actividad de las aves a partir del amanecer. Las evaluaciones se realizaron con el menor silencio posible e identificando y registrando la posición (distancia de la trocha y altura en el bosque) de las aves registrados, visualmente o por oído, a ambos lados de la trocha.

Ocasionalmente fueron utilizadas redes de neblina para capturar aves de sotobosque para facilitar la identificación y registro de especies muy tímidas o de difícil identificación visual.

El estudio se realizó de modo intermitente entre los años 2008 y 2009. Se hizo observaciones sistemáticas y oportunistas en trochas de aproximadamente $2,5 \mathrm{~km}$ en la zona de muestreo. Las trochas se evaluaron desde las primeras horas de la mañana hasta el atardecer.

La abundancia relativa fue estimada empleando nuestros registros continuos. Se ha usado cinco clases de abundancia, de acuerdo con la metodología estandarizada en este tipo de estudios: Común = especies que fueron observadas diariamente y en número considerables (en promedio diez o más aves); Frecuente $=$ especies que han sido vistas diariamente, pero representadas con menos de diez individuos en promedio. Poco común $=$ especies que fueron registradas más de dos veces, pero no diariamente. Rara $=$ especies que fueron observadas u oídas sólo una o dos veces en todo el tiempo de muestreo. Adicionalmente consideramos dos categorías más: Ocasional $=$ especies con un único registro en el área de estudio, de status incierto, pero muy probablemente raras en la zona; y Localmente común o Localmente bastante común = especies que en general no abundan mucho, pero en ciertos lugares muy localizados de la RNAM son bastante abundantes.

La clasificación taxonómica empleada fue la propuesta por Plenge (2011), para los nombres científicos y nombres comunes, de acuerdo con el South American ChecklistCommittee - SACC (Remsen et al., 2011). Para revisar la distribución y rango de las especies se tomó como referencia a Schulenberg et al. (2007).

\section{RESULTADOS}

La avifauna de la cuenca del Misquiyaquillo es muy particular, por la riqueza de especies y por el número de especies de distribución restringida, raras y endémicas.

Como resultado del trabajo realizado en esta zona se ha registrado la presencia de 311especies de aves (Ver Anexo, Lista de aves de la microcuenca del Misquiyaquillo), de las cuales 4 son endémicas para el Perú, una de ellas para el Alto Mayo, 15 son raras o de distribución restringida en Perú, 17 migratorias (14 del Hemisferio norte, y tres del hemisferio sur), y 10 especies categorizadas como amenazadas, por el Perú o por la Unión Internacional para la Conservación de la Naturaleza - IUCN. Adicionalmente hay una lista considerable de especies de alto interés para el aviturismo o birdwaching, que incluyen las citadas y otras 13 especies adicionales (Ver Tablas 1, 2, 3, 4 y 5).

Los dos primeros grupos (especies endémicas para el Perú y especies de distribución restringida) no sólo tienen interés para la conservación, sino que representan el mayor atractivo turístico para la zona. Se ha elaborado mapas preliminares de los lugares donde se puede encontrar con cierta seguridad éstas y otras especies de interés, como insumo para los operadores turísticos.

Las especies citadas en las Tablas 1 y 2 son las de mayor interés para el aviturismo; incluso las especies migratorias (Tabla 3), pues gran parte de los avituristas son originarios de Estados Unidos y Canadá. Adicionalmente a estas especies, en Misquiyaquillo se puede observar con relativa facilidad un número de especies de aves que, aunque son de amplia distribución, son muy difíciles de observar en otras localidades (Tabla 4). También las especies categorizadas en algún grado de amenaza, además de tener importancia para la conservación, son de interés para el aviturismo (Tabla 5). 
Tabla 1. Especies endémicas para el Perú.

$\begin{array}{ll}\text { № } & \text { ESPECIES }\end{array}$

\begin{tabular}{ll}
\hline 1 & Picumnus steindachneri (Carpinterito de Pecho J aspeado, Speckle-chested Piculet) \\
2 & Herpsilochmus parkeri (Hormiguerito de Garganta Ceniza, Ash-throated Antw ren) \\
3 & Zimmerius villarejoi (M oscareta de M ishana, Mishana Tyrannulet) \\
4 & Ramphocelus melanogaster (Tangara de Vientre Negro, Black-bellied Tanager) \\
\hline
\end{tabular}

Tabla 2. Listado de especies raras y de distribución restringida en Perú.

\begin{tabular}{ll}
1 & Touit stictopterus (Periquito de Ala Punteada, Spot-W inged Parrotlet) \\
2 & Caprimulgus maculicaudus (Chotacabras de Cola Punteada, Spot-TailedNightjar) \\
3 & Hydropsalis torquata (Chotacabras de Cola Tijereta, Scissor-tailedNightjar) \\
5 & Synallaxis hypospodia (Cola-Espina de Pecho Cinéreo, Cinereous-breasted Spinetail) \\
6 & Synallaxis cherriei (Cola-Espina de Garganta Castaña, Chestnut-throated spinetail) \\
7 & Hemitriccus striaticollis (Tirano-Todi de Cuello Rayado, Stripe-necked Tody-tyrant) \\
8 & Hemitriccus rufigularis (Tirano-Todi de Garganta Anteada, Buff-throated Tody-Tyrant) \\
9 & M yiophobus roraimae (M osquerito de Roraima; RoraimanFlycatcher) \\
10 & Snow ornis subalaris (Piha de Cola Gris; Gray-tailed Piha) \\
11 & Formicivora rufa (Hormiguerito de Dorso Rojizo, Rusty-backedAntw ren) \\
12 & Laniisoma elegans (Plañidero Elegante, Shrike-like Cotinga) \\
13 & Oryzoborus atrirostris (Semillero de Pico Negro, Black-bellied Seed Finch) \\
14 & Emberizoides herbicola (Sabanero de Cola de Cuña, W edge-tailed Grass-Finch) \\
15 & Tachyphonus phoenicius (Tangara de Hombro Rojo, Red-shouldered Tanager) \\
\hline
\end{tabular}


Tabla 3. Listado de especies migratorias.

№

\begin{tabular}{ll}
\hline 1 & Buteo platypterus (Aguilucho de Ala Ancha, Broad-wingedHawk)* \\
2 & Coccyzus americanus (Cuclillo de Pico Amarillo, Black-billed Cuckoo)* \\
\hline 4 & Contopus virens (Pibí Oriental, Eastern Wood-Pewee)* \\
5 & Contopus cooperi (Pibí Boreal, Olive-sided Flycatcher)* \\
\hline 6 & Contopus sordidulus (Pibí Occidental, Western Wood-Pewee)* \\
\hline 7 & Elaenia parvirostris (Fío Fío de Pico Chico, Small-belled Elaenia)+ \\
8 & Lathrotriccus euleri (M osquerito de Euler, Euler' s Flycatcher)+ \\
\hline 10 & Empidonax alnorum (M osquerito de Alisos, AlderFlycatcher)* \\
\hline 11 & M yiodinastes maculatus (M osquero Rayado, Streaked Flycatcher)+ \\
\hline 12 & Piranga olivacea (Piranga escarlata, Scarlet Taganer)* \\
13 & Dendroica cerulea (Reinita Cerúlea, Ceruleanwabler)* \\
\hline 14 & Dendroica fusca (Reinita de Garganta Naranja, Blackburnian Warbler)* \\
15 & Dendroica striata (Reinita Estriada, Blackpoll Warbler)* \\
\hline 16 & Setophaga ruticilla (Candelita Americana, American Redstart)* \\
\hline 17 & Wilsonia canadensis (Reinita de Canadá, Canada Warbler)* \\
\hline
\end{tabular}

Tabla 4. Listado de especies adicionales de interés para el aviturismo.

\section{№}

1 Eutoxeres condamini (Pico-de-Hoz de Cola Canela; Buff-tailed Sicklebill)

2 Pipreola chlorolepidota (Frutero Garganta de Fuego; Fiery-throated Fruiteater)

3 Pipreola frontalis (Frutero de Pecho Escarlata; Scarlet-breasted Fruiteater)

$4 \quad$ Ampelioides tschudii (Frutero Escamoso; Scaled Fruiteater)

$5 \quad$ Lepidothrix isidorei (Saltarín de Lomo Azul; Blue-rumped Manakin)

6 Epinecrophylla spondionota (Hormiguerito Submontano; Foothill Antw ren)

7 M yrmeciza castanea (Hormiguero de Zimmer; Zimmer' sAntbird)

8 Rhegmatorhina melanosticta (Hormiguero de Cresta Canosa; Hairy-crested Antbird)

9 Synallaxis cherriei (Cola-Espina de Garganta Castaña; Chestnut-throated Spinnetail)

10 Poecilotriccus capitalis (Espatulilla Negra y Blanca; Black-and-w hite Tody-Flycatcher)

11 M achaeropterus pyrocephalus (Saltarín Gorro de Fuego; Fiery-capped M anakin)

12 Schiffornis turdina (ShifornisPardo; Thrush-like Schiffornis)*

13 Tachyphonus rufiventer (Tangara de Cresta Amarilla; Yellow-crested Tanager)

* Aunque se trata de una especie de amplia distribución, la población de M isquiyaquillo corresponde a una forma que parece ser una nueva subespecie. 
Tabla 5. Listado de especies raras y de distribución restringida en Perú.

\begin{tabular}{clll}
\hline \multirow{2}{*}{ No } & \multirow{2}{*}{ ESPECIES } & \multicolumn{2}{c}{ CATEGORIZACIÓN } \\
\cline { 3 - 4 } & & BirdLife/IUCN & \multicolumn{1}{c}{ PERU* } \\
\hline 1 & Aburria aburri & Casi amenazado & Casi amenazado \\
2 & Touit stictopterus & Vulnerable & En peligro \\
3 & Picumnus steindachneri & Vulnerable & En peligro \\
4 & Herpsilochmus parkeri & En peligro & En peligro \\
\hline 5 & Synallaxis cherriei & Casi amenazado & Casi amenazado \\
6 & Hemitriccus rufigularis & Casi amenazado & Casi amenazado \\
7 & Contopus cooperi & Casi amenazado & --------- \\
\hline 8 & Zimmerius villarejoi & Vulnerable & En peligro \\
9 & Dendroica cerulea & Vulnerable & ---------- \\
10 & Pipreola chlorolepidota & Casi amenazado & Casi amenazado \\
\hline * D. S. № 034-2004-AG & &
\end{tabular}

\section{DISCUSIÓN}

Aunque la lista de aves de la microcuenca del Misquiyaquillo todavía no es exhaustiva, es una de las más completas para una localidad en la Región San Martín. En Misquiyaquillo ha sido compilada una lista impresionante de especies endémicas y de distribución restringida en el Perú (Begazo et al., 2001); este hecho, junto con cercanía del sitio a la agradable ciudad de Moyobamba (ambos en la Ruta de Aves del Norte del Perú), a otros sitios cercanos a Tarapoto y a la legendaria Abra Patricia, hacen de Mishquiyaquillo una ideal parada para observadores de aves. El número de especies raras y de distribución restringida registradas hace de Misquiyaquillo también una localidad muy interesante desde el punto también de la conservación.

En Misquiyaquillo uno puede observar con relativa facilidad muchas especies difíciles de ver en otras localidades de la selva del Perú, como Pipreola chlorolepidota, Poecilotriccus capitalis y Micromonacha lanceolata, entre otras, que a pesar de tener amplia distribución son difíciles de encontrar sin realizar una larga y costosa expedición.

Por otro lado, su cercanía con la ciudad de Moyobamba (de la que dista apenas media hora en automóvil), y su ubicación en la llamada Ruta de Aves Noramazónica (Williams et al., 2005) le otorga una ventaja comparativa frente a otras potenciales áreas para observar las especies más raras. Hay diversa oferta de alojamiento en Moyobamba y hay un albergue en la entrada de la quebrada Mishquiyaquillo. Cerca se encuentra otro importante destino turístico, los Baños Termales de San Mateo.
En la región San Martín existen relativamente pocos lugares accesibles desde carretera asfaltada que cuenten con bosque primario entre los 900 y 1600 msnm; esto crea dificultades a las empresas turísticas que promueven la observación de las aves típicas de esta zona.

La especie estrella de Misquiyaquillo es Hepsilochmus parkeri (Hormiguerito de Garganta Ceniza), especie endémica de la región San Martín, ave nombrada en honor al desaparecido Ted Parker (Davis \& O'Neill, 1986). Fue descrita por primera vez de bosque de colinas en Jesús del Monte, una localidad al este de Moyobamba que requiere para llegar de una expedición completay una caminata de unos $15 \mathrm{~km}$. Otro lugar donde puede ser visto es Afluente, en el Bosque de Protección Alto Mayo, pero aquí es infrecuente, por lo que la observación más asequible de esta especie es la cuenca alta del Misquiyaquillo.

A pesar de ser parte del área de conservación ambiental Misquiyacu-Almendra, la microcuenca del Misquiyaquillo no está libre de amenazas. Como casi todas las áreas cercanas a ejes carreteros, esta cuenca ha recibido una fuerte presión en los últimos años por parte de agricultores que se han asentado en el lugar provenientes principalmente de los departamentos de Piura y Cajamarca, los que han adquirido numerosas propiedades de pobladores locales para sembrar cultivos comerciales, especialmente café y cacao.

Con la promover del ecoturismo en la zona, y en particular del aviturismo y el turismo de orquídeas, se espera superar dichos conflictos en el futuro y completar el conocimiento y puesta en valor de la biodiversidad de esta zona. Existen muy buenas 
perspectivas de mejorar la conservación y el aprovechamiento sostenible de la cuenca del Misquiyaquillo articulando esfuerzos en varios frentes, incluyendo la defensa de los bosques remanentes frente a amenazas externas (invasores, cazadores y taladores ilegales, tala indiscriminada de bosques por parte de nuevos propietarios en cumbres de altas pendientes, contaminación de las quebradas por residuos de lavado de café), y la promoción del turismo de naturaleza.

Existen muy buenas perspectivas de que esta visión se haga realidad en un futuro cercano, por cuanto en el lugar están desarrollando actividades de conservación varias instituciones, entre ellas la Municipalidad de Moyobamba, el Proyecto Especial Alto Mayo, La Cooperación Técnica Alemana GIZ, la EPS Moyobamba, y organizaciones particulares, como la Asociación Mishquiyacu, PRONATUR, y Waqanki Orquideario.

\section{BIB LIOGRAFIA CITADA}

Begazo, A. J.; Valqui, T. H.; Sokol, M. ; Langlois, E. 2001. Notes on some birds from central and northern Peru. Cotinga 15: 81-87.

BirdLife International y Conservación Internacional 2005. Áreas importantes para la conservación de las aves en los Andes Tropicales. Quito, Ecuador (Serie de Conservación Birdlife $\mathrm{N}^{\circ} 14$ ).

Clements, J.; N. Shany. 2001. A field guide to the birds of Peru. Ibis Pub. Co., Temecula, Ca.

Davis, T.J.; O'Neill, J.P. 1986. A new especies of antwren (Formicariidae: Herpsilochmus) from Peru, with comments on the systematics of other members of the genus. Wilson Bulletin 98(3): 337352.
Holdridge, L. R. 1967. Ecología basada en zonas de vida. Centro de Estudios Tropicales, San José, Costa Rica. 206 p.

MPM - Municipalidad provincial de Moyobamba. 2002. Expediente Técnico de Creación de la ACM Almendra. Moyobamba, San Martín. 47 pp.

ONERN. 1986. Perfil ambiental del Perú. Lima, Perú. $274 \mathrm{pp}$.

Plenge, M. A. 2011. Lista de las aves de Perú. www.sernanp.gob.pe/sernanp/bpublicaciones.jps ?NroPag=1 (Accesado en enero de 2012)

Ralph, C. H.; Scott ,J. M. 1981. Estimating numbers of terrestrial birds. Studies in Avian Biology No. 6.

Ralph, C. J.; Geupel, G. R.; Pyle, P. ; Martin, T. E. ; De Sante ; D.F. 1993. Handbook of field methods of monitoring landbirds. Pacific Southwest Research Station, California.

Remsen, J. V.; Jaramillo, A.; Nores, M.; Pacheco, J. F.; Robbins, M. B.; Schulenberg, T. S.; Stiles, F. G.; JSilva, . M. C.; Stotz, D. F.; Zimmer, K. J. 2011. A classification of the bird species of South America. www.museum.lsu.edu/ Remsen/SACCBaseline. html (Accesado en febrero 2012).

Ridgely, R. S.; Tudor, G. 1994. The birds of South America. Vol. II, The Suboscine Passerines. Univ. of Texas Press, Austin. 814 pp.

Schulenberg, T. S.; Stotz, D. F.; Lane, D. F.; O'Neill J. P; Parker, T. A. 2007. Birds of Peru. Princeton, NJ: Princeton University Press. 656 pp.

Williams, R.; Álvarez-Alonso, J.; Coppin, L. 2005. Análisis de la factibilidad de la Ruta Aviturística del Norte del Perú con recomendaciones para su desarrollo e implementación. Informe para PROMPERÚ, Lima, 29 pp. 


\section{CLAVE DE LA LISTA DE AVES (LISTA DE AVES DEL ANEXO)}

\section{SÍMBOLOS}

\section{ABUNDANCIA RELATIVA}

O Especie con un único registro en el área de estudio. Estatus incierto, pero muy probablemente rara en la zona.

$\mathrm{R}$ Especie rara, con muy pocos registros. Como mucho observada u oída dos o tres veces en todo el tiempo de muestreo.

U Especie poco común, registrada cada 4-6 días de muestreo en promedio.

F Especie bastante común, registrada casi todos los días de muestreo, aunque en pequeños números.

C Especie común, registrada todos los días, en números significativos (más de 8-10 ejemplares).

$l \mathrm{C}, l \mathrm{~F}$ Localmente común, o localmente bastante común. Se refiere a especies que en general no abundan mucho en el área de estudio, pero que en ciertos lugares muy localizados abundan más.

Los símbolos que hacen referencia a la abundancia relativa precedidos de un " $m$ " son emigrantes, tanto del hemisferio norte como del hemisferio sur.

\section{HÁBITATS}

Bm Bosque primario (con vegetación exuberante y húmeda, cercana a quebradas).

$\mathrm{Bc}$ Bosque primario de colinas altas (con vegetación exuberante y húmeda).

B1 Bosque de laderas con predominancia de especies de Miconeas y 'cascarilla' (Sinchonasp.)

Bn Bosque enano de colinas con vegetación arbustiva dominada por 'indano' y con presencia de gramíneas.

B2 Bosque secundario, en diversas etapas de regeneración, y borde del bosque primario.

Bs Bosques secundarios con predominancia de 'quillosisa', 'ingaina' y otras especies pioneras (partes bajas 900 msnm).

Pc Plantaciones de café bajo sombra.

Ch Chacras y pastizal. Aquí se incluyen hábitats degradados por el hombre, con vegetación herbácea o arbustiva.

Mq Márgenes de quebradas.

$\mathrm{Cb}$ Claros del bosque, provocados naturalmente por la caída de los árboles.

$+\quad$ Más de cuatro tipos de hábitats.

\section{TIPO DE EVIDENCIA.}

V Especie observada.

O Especie de la que se ha oído su vocalización.

Gr Se Cuenta con la grabación de su vocalización (Canto o llamada)

C Espécimen capturado en red de neblina u otra trampa y liberado.

F Espécimen fotografiado.

R Reportado por los pobladores locales (referido solo a especies de fácil identificación). 


\begin{tabular}{|c|c|c|c|c|c|c|c|}
\hline \multicolumn{3}{|c|}{ AVES DE LA SUBCUENCA DEL MISQUIYAQUILLO, MOYOBAMBA, SAN MARTÍN } & \multirow{2}{*}{ 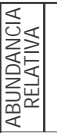 } & \multirow{2}{*}{$\begin{array}{l}\text { PREFERENCIA } \\
\text { DE HABITAT }\end{array}$} & \multirow{2}{*}{ 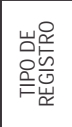 } & \multicolumn{2}{|c|}{$\begin{array}{c}\text { RANGO } \\
\text { ALTITUDINAL } \\
(m, s, n, m)\end{array}$} \\
\hline NOMBRE CIENTÍFICO & NOMBRE EN INGLES & NOMBRE EN ESPAÑOL & & & & \begin{tabular}{l|l|} 
Rango \\
Inicial
\end{tabular} & $\begin{array}{r}\text { Rango } \\
\text { Final }\end{array}$ \\
\hline \multicolumn{8}{|l|}{ Family TINAMIDAE } \\
\hline Tinamus tao & Gray Tinamou & Perdiz Gris & $\mathrm{R}$ & $\mathrm{BP}$ & $\mathrm{V}, 0$ & 1300 & 1400 \\
\hline Crypturellus cinereus & Cinereous Tinamou & Perdiz Cinérea & $\mathrm{U}$ & $\mathrm{BP}, \mathrm{BS}$ & $\mathrm{V}, 0$ & 900 & 1100 \\
\hline Crypturellus soui & Little Tinamou & Perdiz Chica & $\mathrm{U}$ & $\mathrm{BP}, \mathrm{BS}$ & $\mathrm{V}, 0$ & 1000 & 1200 \\
\hline Crypturellus tataupa & Tataupa Tinamou & Perdiz Tataupá & $\mathrm{U}$ & $P C, A D$ & $\mathrm{~V}, 0$ & 1000 & 1200 \\
\hline \multicolumn{8}{|l|}{ Family CRACIDAE } \\
\hline Penelope jacquacu & Spix's Guan & Pava de Spix & $U$ & $\mathrm{BP}$ & $V, 0$ & 1100 & 1300 \\
\hline Aburria aburri & Wattled Guan & Pava Carunculada & $\mathrm{U}$ & $\mathrm{BP}$ & $\mathrm{V}, 0$ & 1200 & 1620 \\
\hline Ortalis guttata & Speckled Chachalaca & Chachalaca Jaspeada & $\mathrm{F}$ & $\mathrm{BP}, \mathrm{BS}, \mathrm{PC}$ & $\mathrm{V}, 0$ & 900 & 1620 \\
\hline \multicolumn{8}{|l|}{ Family ODONTOPHORIDAE } \\
\hline Odontophorus gujanensis & Marbled Wood-Quail & Codorniz de Cara Roja & $\mathrm{F}$ & $\mathrm{BP}$ & $\mathrm{V}, 0$ & 1000 & 1620 \\
\hline Odontophorus speciosus & Rufous-breasted Wood-Quail & Codorniz de Pecho Rufo & $U$ & $\mathrm{BP}$ & $\mathrm{V}, 0$ & 1200 & 1620 \\
\hline \multicolumn{8}{|l|}{ Family ARDEIDAE } \\
\hline Tigrisoma lineatum & Rufescent Tiger-Heron & Garza-Tigre Colorada & $\mathrm{R}$ & $\mathrm{BP}, \mathrm{BS}$ & $\mathrm{V}$ & 900 & 1100 \\
\hline Butorides striata & Striated Heron & Garcita Estriada & $\mathrm{R}$ & $\mathrm{AD}$ & $\mathrm{V}$ & 900 & 1000 \\
\hline Bubulcus ibis & Cattle Egret & Garcita Bueyera & $\mathrm{F}$ & $\mathrm{AD}$ & $\mathrm{V}$ & 900 & 1100 \\
\hline \multicolumn{8}{|l|}{ Family CATHARTIDAE } \\
\hline Cathartes aura & Turkey Vulture & Gallinazo de Cabeza Roja & $\mathrm{F}$ & $\mathrm{BP}, \mathrm{BS}, \mathrm{BL}, \mathrm{PC}, \mathrm{AD}$ & $\mathrm{V}$ & 900 & 1620 \\
\hline Coragyps atratus & Black Vulture & Gallinazo de Cabeza Negra & $\mathrm{F}$ & $\mathrm{BP}, \mathrm{BS}, \mathrm{BL}, \mathrm{PC}, \mathrm{AD}$ & v & 900 & 1620 \\
\hline \multicolumn{8}{|l|}{ Family ACCIPITRIDAE } \\
\hline Leptodon cayanensis & Gray-headed Kite & Elanio de Cabeza Gris & $\mathrm{R}$ & BS & $\mathrm{V}$ & 900 & 1000 \\
\hline Elanoides forficatus & Swallow-tailed Kite & Elanio Tijereta & C & $\mathrm{BP}, \mathrm{BS}, \mathrm{BL}, \mathrm{PC}, \mathrm{AD}$ & $\mathrm{V}, 0$ & 900 & 1620 \\
\hline Harpagus bidentatus & Double-toothed Kite & Elanio Bidentado & U & $\mathrm{BP}, \mathrm{BS}$ & $\mathrm{V}, 0$ & 1100 & 1300 \\
\hline Ictinia plumbea & Plumbeous Kite & Elanio Plomizo & U & $\mathrm{PC}$ & $\mathrm{V}$ & 1400 & 1500 \\
\hline Leucopternis albicollis & White Hawk & Gavilán Blanco & U & $\mathrm{BP}, \mathrm{BL}$ & $\mathrm{V}$ & 1400 & 1500 \\
\hline Buteo magnirostris & Roadside Hawk & Aguilucho Caminero & $\mathrm{F}$ & $\mathrm{BP}, \mathrm{BS}, \mathrm{PC}, \mathrm{AD}$ & $\mathrm{V}, 0$ & 900 & 1620 \\
\hline Buteo platypterus (NB) & Broad-winged Hawk & Aguilucho de Ala Ancha & $\mathrm{mU}$ & $\mathrm{PC}$ & $\mathrm{V}, 0$ & 900 & 1500 \\
\hline Buteo brachyurus & Short-tailed Hawk & Aguilucho de Cola Corta & \begin{tabular}{|l|l|}
$u$ \\
\end{tabular} & $\mathrm{BP}, \mathrm{BS}, \mathrm{BL}$ & $\mathrm{V}, 0$ & 1100 & 1500 \\
\hline \multicolumn{8}{|l|}{ Family FALCONIDAE } \\
\hline Daptrius ater & Black Caracara & Caracara Negro & $\mathrm{U}$ & $\mathrm{BP}, \mathrm{BS}$ & $\mathrm{V}, 0$ & 900 & 1200 \\
\hline Falco deiroleucus & Orange-breasted Falcon & Halcón de Pecho Naranja & $\mathrm{U}$ & $\mathrm{PC}$ & $\mathrm{V}$ & 1300 & 1500 \\
\hline \multicolumn{8}{|l|}{ Family RALLIDAE } \\
\hline Anurolimnas viridis & Russet-crowned Crake & Gallineta de Corona Rufa & $\mathrm{F}$ & $\mathrm{BL}, \mathrm{AD}$ & $\mathrm{V}, 0$ & 900 & 1500 \\
\hline Family COLUMBIDAE & & & & & & & \\
\hline Columbina talpacoti & Ruddy Ground-Dove & Tortolita Rojiza & $\mathrm{C}$ & $\mathrm{AD}$ & $\mathrm{V}, 0$ & 900 & 1000 \\
\hline Claravis pretiosa & Blue Ground-Dove & Tortolita Azul & $\mathrm{F}$ & $\mathrm{BS}, \mathrm{PC}, \mathrm{AD}$ & $\mathrm{V}, 0$ & 900 & 1400 \\
\hline Patagioenas speciosa & Scaled Pigeon & Paloma Escamosa & $\mathrm{F}$ & $\mathrm{BL}$ & $\mathrm{V}, 0$ & 1200 & 1620 \\
\hline Patagioenas plumbea & Plumbeous Pigeon & Paloma Plomiza & $\mathrm{F}$ & $\mathrm{BP}, \mathrm{BS}$ & $\mathrm{V}, 0$ & 1200 & 1620 \\
\hline Leptotila verreauxi & White-tipped Dove & Paloma de Puntas Blancas & $\mathrm{F}$ & $\mathrm{BS}, \mathrm{PC}, \mathrm{AD}$ & $\mathrm{V}, 0$ & 900 & 1620 \\
\hline Geotrygon frenata & White-throated Quail-Dove & Paloma-Perdiz de Garganta Blanca & $\mathrm{F}$ & $\mathrm{BP}$ & $\mathrm{V}, 0$ & 1300 & 1620 \\
\hline Geotrygon montana & Ruddy Quail-Dove & Paloma-Perdiz Rojiza & $\mathrm{U}$ & $\mathrm{BP}, \mathrm{BS}, \mathrm{PC}$ & $\mathrm{V}, 0$ & 1000 & 1300 \\
\hline Family PSITTACIDAE & & & & & & & \\
\hline Aratinga leucophthalma & White-eyed Parakeet & Cotorra de Ojo Blanco & $\mathrm{C}$ & $\mathrm{BS}, \mathrm{PC}$ & $\mathrm{V}, 0$ & 900 & 1620 \\
\hline Forpus xanthopterygius & Blue-winged Parrotlet & Periquito de Ala Azul & $\mathrm{U}$ & BS & $V, 0$ & 900 & 1000 \\
\hline Brotogeris cyanoptera & Cobalt-winged Parakeet & Perico de Ala Cobalto & $\mathrm{C}$ & $\mathrm{BS}, \mathrm{PC}$ & $\mathrm{V}, 0$ & 900 & 1200 \\
\hline Touit stictopterus & Spot-winged Parrotlet & Periquito de Ala Punteada & $\mathrm{U}$ & $\mathrm{BP}$ & $\mathrm{V}, 0$ & 1200 & 1620 \\
\hline Pionus menstruus & Blue-headed Parrot & Loro de Cabeza Azúl & $\mathrm{F}$ & $\mathrm{BP}, \mathrm{BS}, \mathrm{PC}$ & $\mathrm{V}, 0$ & 900 & 1620 \\
\hline Amazona mercenarius & Scaly-naped Parrot & Loro de Nuca Escamosa & $\mathrm{U}$ & $\mathrm{BP}$ & $\mathrm{V}, 0$ & 1500 & 1620 \\
\hline Family CUCULIDAE & & & & & & & \\
\hline Piaya cayana & Squirrel Cuckoo & Cuco Ardilla & $\mathrm{C}$ & $\mathrm{BP}, \mathrm{BS}, \mathrm{BL}, \mathrm{PC}$ & $\mathrm{V}, 0$ & 900 & 1620 \\
\hline Coccyzus americanus (NB) & Yellow-billed Cuckoo & Cuclillo de Pico Amarillo & $\mathrm{mR}$ & $\mathrm{BS}, \mathrm{PC}$ & $\mathrm{V}, 0$ & 1000 & 1100 \\
\hline Crotophaga ani & Smooth-billed Ani & Garrapatero de Pico Liso & $\mathrm{C}$ & $\mathrm{AD}$ & $\mathrm{V}, 0$ & 900 & 1400 \\
\hline Family STRIGIDAE & & & & & & & \\
\hline Megascops choliba & Tropical Screech-0wl & Lechuza Tropical & $\mathrm{U}$ & $\mathrm{BS}, \mathrm{PC}$ & $\mathrm{V}, 0$ & 900 & 1200 \\
\hline Megascops ingens & Rufescent Screech-0wl & Lechuza Rojiza & $\mathrm{R}$ & $\mathrm{BP}$ & $\mathrm{V}, 0$ & 1000 & 1300 \\
\hline Megascops guatemalae & Vermiculated Screech-0wl & Lechuza Vermiculada & $\mathrm{F}$ & $\mathrm{BP}$ & $\mathrm{V}, 0$ & 1200 & 1620 \\
\hline Pulsatrix melanota & Band-bellied Owl & Búho de Vientre Bandeado & $\mathrm{U}$ & $\mathrm{BP}$ & $\mathrm{V}, 0$ & 900 & 1620 \\
\hline Ciccaba huhula & Black-banded Owl & Búho Negro Bandeado & U & $\mathrm{BP}, \mathrm{BS}$ & $V, 0$ & 900 & 1000 \\
\hline Glaucidium brasilianum & Ferruginous Pygmy-Owl & Lechucita Ferruginosa & $\mathrm{F}$ & $\mathrm{BS}, \mathrm{PC}$ & $V, 0$ & 900 & 1500 \\
\hline Family NYCTIBIIDAE & & & & & & & \\
\hline Nyctibius griseus & Common Potoo & Nictibio Común & $\mathrm{F}$ & $\mathrm{BP}$ & 0 & 1200 & 1620 \\
\hline Nyctibius bracteatus & Rufous Potoo & Nictibio Rufo & $\mathrm{R}$ & $\mathrm{BP}$ & $\mathrm{V}, 0$ & 1100 & 1200 \\
\hline
\end{tabular}




\begin{tabular}{|c|c|c|c|c|c|c|c|}
\hline \multicolumn{3}{|c|}{ AVES DE LA SUBCUENCA DEL MISQUIYAQUILLO, MOYOBAM BA, SAN MARTÍN } & \multirow{2}{*}{ 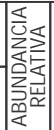 } & \multirow{2}{*}{$\begin{array}{l}\text { PREFERENCIA } \\
\text { DE HABITAT }\end{array}$} & \multirow{2}{*}{ 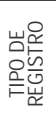 } & \multicolumn{2}{|c|}{$\begin{array}{c}\text { RANGO } \\
\text { ALTITUDINAL } \\
(\mathrm{m}, \mathrm{s}, \mathrm{n}, \mathrm{m})\end{array}$} \\
\hline NOMBRE CIENTÍFICO & NOMBRE EN INGLES & NOMBRE EN ESPAÑOL & & & & $\begin{array}{l}\text { Rango } \\
\text { Inicial }\end{array}$ & $\begin{array}{r}\text { Rango } \\
\text { Final }\end{array}$ \\
\hline \multicolumn{8}{|l|}{ Family CAPRIMULGIDAE } \\
\hline Nyctidromus albicollis & Common Pauraque & Chotacabras Común & $\mathrm{F}$ & $\mathrm{PC}, \mathrm{AD}$ & $\mathrm{V}, 0$ & 900 & 1200 \\
\hline Nyctiphrynus ocellatus & Ocellated Poorwill & Chotacabras 0celado & $\mathrm{U}$ & $\mathrm{BS}, \mathrm{BP}$ & $\mathrm{V}, 0$ & 940 & 1200 \\
\hline Antrostomus rufus $(\mathrm{H})$ & Rufous Nightjar & Chotacabras Rufo & $\mathrm{U}$ & $\mathrm{BS}, \mathrm{BL}$ & $\mathrm{V}$ & 1000 & 1350 \\
\hline Caprimulgus maculicaudus & Spot-tailed Nightjar & Chotacabras de Cola Punteada & $\mathrm{F}$ & $A D$ & $\mathrm{~V}, 0$ & 900 & 1000 \\
\hline Caprimulgus parvulus (NB) & Little Nightjar & Chotacabras Chico & $\mathrm{mR}$ & $B S, A D$ & $\mathrm{~V}, 0$ & 940 & 1100 \\
\hline Caprimulgus nigrescens & Blackish Nightjar & Chotacabras Negruzco & $\mathrm{U}$ & $\mathrm{BS}, \mathrm{AD}$ & $\mathrm{V}, 0$ & 1400 & 1620 \\
\hline Hydropsalis torquata & Scissor-tailed Nightjar & Chotacabras de Cola Tijereta & 0 & $\mathrm{BL}$ & $\mathrm{V}, \mathrm{R}$ & 1200 & 1450 \\
\hline \multicolumn{8}{|l|}{ Family APODIDAE } \\
\hline Streptoprocne zonaris & White-collared Swift & Vencejo de Collar Blanco & U & $B S, B L, P C, A D$ & $\mathrm{~V}$ & 900 & 1620 \\
\hline Tachornis squamata & Fork-tailed Palm-Swift & Vencejo Tijereta de Palmeras & $\mathrm{U}$ & $A D$ & $\mathrm{~V}$ & 900 & 1000 \\
\hline \multicolumn{8}{|l|}{ Family TROCHILIDAE } \\
\hline Florisuga mellivora & White-necked Jacobin & Colibrí de Nuca Blanca & $\mathrm{F}$ & $\mathrm{PC}$ & $\mathrm{V}$ & 900 & 1200 \\
\hline Eutoxeres condamini & Buff-tailed Sicklebill & Pico-de-Hoz de Cola Canela & $\mathrm{F}$ & $\mathrm{BP}, \mathrm{BS}$ & $\mathrm{V}$ & 1000 & 1620 \\
\hline Glaucis hirsutus & Rufous-breasted Hermit & Ermitaño de Pecho Canela & $\mathrm{U}$ & $\mathrm{PC}$ & $\mathrm{V}$ & 900 & 1000 \\
\hline Phaethornis atrimentalis & Black-throated Hermit & Ermitaño de Garganta Negra & $\mathrm{F}$ & $\mathrm{BP}, \mathrm{BS}, \mathrm{PC}$ & $\mathrm{V}$ & 900 & 1100 \\
\hline Phaethornis ruber & Reddish Hermit & Ermitaño Rojizo & $\mathrm{F}$ & $\mathrm{BP}, \mathrm{BS}, \mathrm{PC}$ & V & 1200 & 1400 \\
\hline Phaethornis guy & Green Hermit & Ermitaño Verde & $\mathrm{F}$ & $\mathrm{BP}, \mathrm{BS}$ & V & 1000 & 1620 \\
\hline Phaethornis malaris (supercillosus) & Great-billed (Long.tailed) Hermit & Ermitaño de (pico Grande) Cola Larga & $\mathrm{F}$ & $\mathrm{BP}, \mathrm{BS}, \mathrm{PC}$ & $\mathrm{V}$ & 900 & 1300 \\
\hline Doryfera johannae & Blue-fronted Lancebill & Pico-Lanza de Frente Azul & $\mathrm{U}$ & $\mathrm{BS}$ & $\mathrm{V}$ & 900 & 1200 \\
\hline Colibri delphinae & Brown Violetear & Oreja-Violeta Parda & $\mathrm{U}$ & $\mathrm{BP}, \mathrm{BS}$ & $\mathrm{V}, 0$ & 1200 & 1620 \\
\hline Colibri coruscans & Sparkling Violetear & Oreja-Violeta de Vientre Azul & $\mathrm{F}$ & $\mathrm{BL}, \mathrm{PC}$ & $\mathrm{V}, 0$ & 900 & 1620 \\
\hline Heliothryx auritus & Black-eared Fairy & Colibrí-Hada de Oreja Negra & $\mathrm{U}$ & $\mathrm{BP}, \mathrm{BS}$ & $\mathrm{V}$ & 1000 & 1300 \\
\hline Discosura popelairii & Wire-crested Thorntail & Cola-Cerda Crestado & $\mathrm{R}$ & BS & $\mathrm{V}$ & 1400 & 1500 \\
\hline Lophornis delattrei & Rufous-crested Coquette & Coqueta de Cresta Rufa & $\mathrm{F}$ & $\mathrm{BS}, \mathrm{PC}$ & V & 1000 & 1500 \\
\hline Lophornis chalybeus & Festive Coquette & Coqueta Verde & $\mathrm{U}$ & $\mathrm{BS}, \mathrm{PC}$ & V & 1000 & 1100 \\
\hline Heliomaster longirostris & Long-billed Starthroat & Colibrí de Pico Largo & $\mathrm{F}$ & $\mathrm{PC}$ & $\mathrm{V}$ & 900 & 1000 \\
\hline Chaetocercus mulsant & White-bellied Woodstar & Estrellita de Vientre Blanco & $\mathrm{U}$ & $\mathrm{PC}$ & $\mathrm{V}$ & 1000 & 1500 \\
\hline Chaetocercus bombus & Little Woodstar & Estrellita Chica & $\mathrm{U}$ & $\mathrm{BS}$ & $\mathrm{V}$ & 900 & 1400 \\
\hline Calliphlox amethystina & Amethyst Woodstar & Estrellita Amatista & $\mathrm{U}$ & $\mathrm{BS}, \mathrm{PC}$ & $\mathrm{V}$ & 900 & 1200 \\
\hline Chlorostilbon mellisugus & Blue-tailed Emerald & Esmeralda de Cola Azul & $\mathrm{F}$ & $\mathrm{BL}, \mathrm{AD}$ & $\mathrm{V}$ & 900 & 1500 \\
\hline Klais guimeti & Violet-headed Hummingbird & Colibrí de Cabeza Violeta & U & $\mathrm{BP}, \mathrm{BS}$ & $\mathrm{V}$ & 1000 & 1500 \\
\hline Campylopterus largipennis & Gray-breasted Sabrewing & Ala-de-Sable de Pecho Gris & U & $\mathrm{BP}, \mathrm{BS}, \mathrm{PC}$ & $\mathrm{V}, \mathrm{C}$ & 900 & 1620 \\
\hline Thalurania furcata & Fork-tailed Woodnymph & Ninfa de Cola Ahorquillada & $\mathrm{F}$ & $\mathrm{BP}, \mathrm{BS}, \mathrm{PC}$ & $\mathrm{V}, \mathrm{C}$ & 1000 & 1400 \\
\hline Taphrospilus hypostictus & Many-spotted Hummingbird & Colibrí Multipunteado & $\mathrm{F}$ & $\mathrm{BP}$ & $\mathrm{V}$ & 1200 & 1400 \\
\hline Amazilia lactea & Sapphire-spangled Emerald & Colibrí de Pecho Zafiro & U & $A D$ & $\mathrm{~V}$ & 900 & 1000 \\
\hline Chrysuronia oenone & Golden-tailed Sapphire & Zafiro de Cola Dorada & $\mathrm{F}$ & $\mathrm{PC}$ & V, C & 900 & 1500 \\
\hline Hylocharis cyanus & White-chinned Sapphire & Zafiro de Barbilla Blanca & $\mathrm{F}$ & BS & $\mathrm{V}$ & 900 & 1000 \\
\hline \multicolumn{8}{|l|}{ Family TROGONIDAE } \\
\hline Pharomachrus antisianus & Crested Quetzal & Quetzal Crestado & $\mathrm{R}$ & $\mathrm{BP}$ & $\mathrm{V}$ & 1300 & 1620 \\
\hline Trogon viridis & Green-backed Trogon & Trogón de Dorso Verde & $\mathrm{F}$ & $\mathrm{BP}, \mathrm{BS}, \mathrm{PC}$ & $\mathrm{V}, 0$ & 1000 & 1300 \\
\hline Trogon ramonianus & Amazonian Trogon & Trogón Amazónico & $\mathrm{U}$ & $\mathrm{BS}, \mathrm{BS}$ & $\mathrm{V}, 0$ & 900 & 1100 \\
\hline Trogon curucui & Blue-crowned Trogon & Trogón de Corona Azul & $\mathrm{F}$ & $\mathrm{BP}, \mathrm{BS}$ & $\mathrm{V}, 0$ & 1100 & 1500 \\
\hline Trogon collaris & Collared Trogon & Trogón Acollarado & $\mathrm{F}$ & $\mathrm{BP}, \mathrm{BS}, \mathrm{PC}$ & $\mathrm{V}, 0$ & 1000 & 1620 \\
\hline \multicolumn{8}{|l|}{ Family ALCEDINIDAE } \\
\hline Chloroceryle americana & Green Kingfisher & Martín Pescador Verde & \begin{tabular}{l|l}
$\mathrm{R}$ & \\
\end{tabular} & $\mathrm{BP}, \mathrm{BS}$ & $\mathrm{V}$ & 1000 & 1200 \\
\hline \multicolumn{8}{|l|}{ Family MOMOTIDAE } \\
\hline Electron platyrhynchum & Broad-billed Motmot & Relojero de Pico Ancho & $\mathrm{F}$ & $\mathrm{BP}$ & $V, 0$ & 1100 & 1620 \\
\hline Baryphthengus martii & Rufous Motmot & Relojero Rufo & $\mathrm{F}$ & $\mathrm{BP}, \mathrm{BS}$ & $\mathrm{V}, 0$ & 1000 & 1300 \\
\hline \multicolumn{8}{|l|}{ Family GALBULIDAE } \\
\hline Galbula albirostris & Yellow-billed Jacamar & Jacamar de Pico Amarillo & U & $\mathrm{BS}$ & $\mathrm{V}, 0$ & 900 & 1000 \\
\hline Galbula cyanescens & Bluish-fronted Jacamar & Jacamar de Frente Azulada & $\mathrm{F}$ & $\mathrm{BP}, \mathrm{BS}, \mathrm{PC}$ & $\mathrm{V}, 0$ & 1000 & 1200 \\
\hline \begin{tabular}{|l|} 
Jacamerops aureus \\
\end{tabular} & Great Jacamar & Jacamar Grande & $R$ & $\mathrm{BP}$ & $\mathrm{V}, 0$ & 1000 & 1200 \\
\hline \multicolumn{8}{|l|}{ Family BUCCONIDAE } \\
\hline Nystalus striolatus & Striolated Puffbird & Buco Estriolado & $\mathrm{U}$ & $\mathrm{BP}, \mathrm{BL}$ & $\mathrm{V}, 0$ & 1200 & 1500 \\
\hline Micromonacha lanceolata & Lanceolated Monklet & Monjecito Lanceolado & $\mathrm{U}$ & $\mathrm{BP}, \mathrm{BS}$ & $\mathrm{V}, 0$ & 1000 & 1300 \\
\hline Nonnula ruficapilla & Rufous-capped Nunlet & Monjita de Gorro Rufo & $\mathrm{U}$ & $\mathrm{BP}, \mathrm{BS}$ & $\mathrm{V}$ & 1200 & 1500 \\
\hline Monasa morphoeus & White-fronted Nunbird & Monja de Frente Blanca & $\mathrm{U}$ & $\mathrm{BP}, \mathrm{BL}$ & $\mathrm{V}, 0$ & 1000 & 1200 \\
\hline \multicolumn{8}{|l|}{ Family CAPITONIDAE } \\
\hline Capito auratus & Gilded Barbet & Barbudo Brilloso & C & $\mathrm{BS}, \mathrm{PC}$ & $\mathrm{V}, 0$ & 900 & 1500 \\
\hline Eubucco versicolor & Versicolored Barbet & Barbudo Versicolor & $\mathrm{F}$ & $\mathrm{BP}, \mathrm{BS}$ & $\mathrm{V}$ & 1300 & 1620 \\
\hline
\end{tabular}




\begin{tabular}{|c|c|c|c|c|c|c|c|}
\hline \multicolumn{3}{|c|}{ AVES DE LA SUBCUENCA DEL MISQUIYAQUILLO, MOYOBAM BA, SAN MARTÍN } & \multirow{2}{*}{ 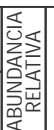 } & \multirow{2}{*}{$\begin{array}{l}\text { PREFERENCIA } \\
\text { DE HABITAT }\end{array}$} & \multirow{2}{*}{ 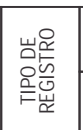 } & \multicolumn{2}{|c|}{$\begin{array}{c}\text { RANGO } \\
\text { ALTIIUDINAL } \\
(\mathrm{m} . \mathrm{s} . \mathrm{n} . \mathrm{m})\end{array}$} \\
\hline NOMBRE CIENTÍFICO & NOMBRE EN INGLES & NOMBRE EN ESPAÑOL & & & & \begin{tabular}{l|} 
Rango \\
Inicial
\end{tabular} & $\begin{array}{l}\text { Rango } \\
\text { Final }\end{array}$ \\
\hline \multicolumn{8}{|l|}{ Family RAMPHASTIDAE } \\
\hline Ramphastos vitellinus & Channel-billed Toucan & Tucán de Pico Acanalado & $\mathrm{F}$ & $\mathrm{BP}, \mathrm{BL}$ & $\mathrm{V}, 0$ & 1000 & 1620 \\
\hline Aulacorhynchus derbianus & Chestnut-tipped Toucanet & Tucancillo de Puntas Castañas & $\mathrm{F}$ & $\mathrm{BP}$ & $\mathrm{V}, 0$ & 1500 & 1620 \\
\hline Selenidera reinwardtii & Golden-collared Toucanet & Tucancillo de Collar Dorado & $\mathrm{F}$ & $\mathrm{BP}$ & $\mathrm{V}, 0$ & 1000 & 1500 \\
\hline Pteroglossus inscriptus & Lettered Araçari & Arasari Letreado & $\mathrm{F}$ & $\mathrm{BP}, \mathrm{BS}, \mathrm{PC}$ & $\mathrm{V}, 0$ & 1000 & 1400 \\
\hline Pteroglossus castanotis & Chestnut-eared Araçari & Arasari de Oreja Castaña & $\mathrm{F}$ & $\mathrm{BP}, \mathrm{BS}, \mathrm{PC}$ & $\mathrm{V}, 0$ & 900 & 1300 \\
\hline Pteroglossus azara & Ivory-billed Araçari & Arasari de Pico Marfil & $\mathrm{R}$ & $\mathrm{BP}$ & $\mathrm{V}$ & 1100 & 1200 \\
\hline \multicolumn{8}{|l|}{ Family PICIDAE } \\
\hline Picumnus lafresnayi & Lafresnaye's Piculet & Carpinterito de Lafresnaye & $\mathrm{F}$ & $\mathrm{BP}, \mathrm{BS}, \mathrm{PC}$ & $\mathrm{V}, 0$ & 900 & 1200 \\
\hline Picumnus steindachneri (E) & Speckle-chested Piculet & Carpinterito de Pecho Jaspeado & $\mathrm{U}$ & $\mathrm{BS}, \mathrm{PC}$ & $\mathrm{V}, 0$ & 1400 & 1620 \\
\hline Melanerpes cruentatus & Yellow-tufted Woodpecker & Carpintero de Penacho Amarillo & $\mathrm{F}$ & $\mathrm{BS}, \mathrm{PC}$ & $\mathrm{V}, 0$ & 1000 & 1500 \\
\hline Picoides fumigatus & Smoky-brown Woodpecker & Carpintero Pardo & U & $\mathrm{BP}$ & $\mathrm{V}$ & 1500 & 1620 \\
\hline Veniliornis passerinus & Little Woodpecker & Carpintero Chico & $\mathrm{F}$ & $\mathrm{BS}, \mathrm{PC}$ & $V, 0$ & 900 & 1000 \\
\hline \begin{tabular}{|l} 
Veniliornis affinis \\
\end{tabular} & Red-stained Woodpecker & Carpintero Teñido de Rojo & U & $\mathrm{BL}$ & $V, 0$ & 1100 & 1400 \\
\hline Piculus leucolaemus & White-throated Woodpecker & Carpintero de Garganta Blanca & U & $\mathrm{PC}$ & $\mathrm{V}$ & 1400 & 1500 \\
\hline Piculus chrysochloros & Golden-green Woodpecker & Carpintero Verde y Dorado & U & $\mathrm{BP}$ & $V, 0$ & 1400 & 1500 \\
\hline Colaptes rubiginosus & Golden-olive Woodpecker & Carpintero Olivo y Dorado & $U$ & $\mathrm{BP}$ & $V, 0$ & 1300 & 1620 \\
\hline Colaptes punctigula & Spot-breasted Woodpecker & Carpintero de Pecho Punteado & $U$ & BS & $V, 0$ & 900 & 1000 \\
\hline Dryocopus lineatus & Lineated Woodpecker & Carpintero Lineado & U & $\mathrm{BP}, \mathrm{BS}, \mathrm{PC}$ & $\mathrm{V}, 0$ & 1000 & 1500 \\
\hline Campephilus rubricollis & Red-necked Woodpecker & Carpintero de Cuello Rojo & $\mathrm{R}$ & $\mathrm{BP}$ & $\mathrm{V}$ & 1200 & 1600 \\
\hline \multicolumn{8}{|l|}{ Family THAMNOPHILIDAE } \\
\hline Cymbilaimus lineatus & Fasciated Antshrike & Batará Lineado & U & $\mathrm{BP}$ & $\mathrm{V}, 0$ & 1100 & 1300 \\
\hline Taraba major & Great Antshrike & Batará Grande & $\mathrm{F}$ & BS & $\mathrm{V}, 0$ & 1000 & 1450 \\
\hline Thamnophilus doliatus & Barred Antshrike & Batará Barrado & $\mathrm{F}$ & BS & $\mathrm{V}, 0$ & 900 & 1000 \\
\hline Thamnophilus tenuepunctatus & Lined Antshrike & Batará Listado & $\mathrm{F}$ & $\mathrm{BP}, \mathrm{BS}$ & $\mathrm{V}, 0$ & 1300 & 1620 \\
\hline Thamnophilus schistaceus & Plain-winged Antshrike & Batará de Ala Llana & $\mathrm{F}$ & $\mathrm{BP}, \mathrm{BS}, \mathrm{PC}$ & $\mathrm{V}, 0$ & 1000 & 1300 \\
\hline Dysithamnus mentalis & Plain Antvireo & Batarito de Cabeza Gris & $\mathrm{F}$ & $\mathrm{BP}$ & $\mathrm{V}, 0$ & 1000 & 1620 \\
\hline Epinecrophylla spodionota & Foothill Antwren & Hormiguerito Submontano & $\mathrm{F}$ & $\mathrm{BP}$ & $\mathrm{V}, 0$ & 1000 & 1500 \\
\hline Epinecrophylla ornata & Ornate Antwren & Hormiguerito Adornado & $\mathrm{F}$ & BP, BS & $\mathrm{V}$ & 1200 & 1500 \\
\hline Myrmotherula longicauda & Stripe-chested Antwren & Hormiguerito de Pecho Listado & $\mathrm{F}$ & $\mathrm{BS}, \mathrm{PC}$ & $\mathrm{V}, 0$ & 1000 & 1400 \\
\hline Myrmotherula axillaris & White-flanked Antwren & Hormiguerito de Flanco Blanco & $\mathrm{F}$ & $\mathrm{BP}$ & $\mathrm{V}, 0$ & 1000 & 1450 \\
\hline Herpsilochmus parkeri (E) & Ash-throated Antwren & Hormiguerito de Garganta Ceniza & $\mathrm{F}$ & $\mathrm{BP}$ & $\mathrm{V}, 0, \mathrm{Gr}$ & 1400 & 1620 \\
\hline Herpsilochmus axillaris & Yellow-breasted Antwren & Hormiguerito de Pecho Amarillo & $\mathrm{F}$ & $\mathrm{BP}$ & $\mathrm{V}, 0$ & 1100 & 1400 \\
\hline Formicivora rufa & Rusty-backed Antwren & Hormiguerito de Dorso Rojizo & $\mathrm{U}$ & $\mathrm{BL}$ & $\mathrm{V}, 0$ & 1300 & 1400 \\
\hline Hypocnemis peruviana & Peruvian Warbling-Antbird & Hormiguero Peruano & $\mathrm{F}$ & $\mathrm{BP}, \mathrm{BS}, \mathrm{PC}$ & $\mathrm{V}, 0$ & 1000 & 1500 \\
\hline Cercomacra cinerascens & Gray Antbird & Hormiguero Gris & $\mathrm{U}$ & $\mathrm{BP}$ & $\mathrm{V}, 0, \mathrm{Gr}$ & 1000 & 1300 \\
\hline Cercomacra nigrescens & Blackish Antbird & Hormiguero Negruzco & $\mathrm{F}$ & $\mathrm{BP}, \mathrm{BS}$ & $\mathrm{V}, 0, \mathrm{Gr}$ & 1000 & 1300 \\
\hline Myrmoborus leucophrys & White-browed Antbird & Hormiguero de Ceja Blanca & $\mathrm{F}$ & $\mathrm{BP}, \mathrm{BS}$ & $\mathrm{V}, 0$ & 1000 & 1400 \\
\hline Schistocichla leucostigma & Spot-winged Antbird & Hormiguero de Ala Moteada & $\mathrm{F}$ & $\mathrm{BP}$ & $\mathrm{V}, 0$ & 1000 & 1300 \\
\hline Myrmeciza castanea & Zimmer's Antbird & Hormiguero de Zimmer & $\mathrm{F}$ & $\mathrm{BL}$ & $\mathrm{V}$ & 1100 & 1300 \\
\hline Pithys albifrons & White-plumed Antbird & Hormiguero de Plumón Blanco & $\mathrm{U}$ & $\mathrm{BP}$ & $\mathrm{V}, 0$ & 1000 & 1350 \\
\hline Rhegmatorhina melanosticta & Hairy-crested Antbird & Hormiguero de Cresta Canosa & U & $\mathrm{BP}$ & V & 1000 & 1350 \\
\hline Hylophylax naevius & Spot-backed Antbird & Hormiguero de Dorso Moteado & $\mathrm{F}$ & $\mathrm{BP}$ & $\mathrm{V}, 0$ & 1000 & 1400 \\
\hline Willisornis poecilinotus & Scale-backed Antbird & Hormiguero de Dorso Escamoso & $\mathrm{U}$ & $\mathrm{BP}$ & $\mathrm{V}, 0$ & 1000 & 1350 \\
\hline \multicolumn{8}{|l|}{ Family GRALLARIIDAE } \\
\hline Grallaria guatimalensis & Scaled Antpitta & Tororoi Escamoso & $\mathrm{F}$ & $\mathrm{BP}$ & $\mathrm{V}, 0$ & 1300 & 1500 \\
\hline \multicolumn{8}{|l|}{ Family RHINOCRYPTIDAE } \\
\hline Scytalopus atratus & White-crowned Tapaculo & Tapaculo de Corona Blanca & $\mathrm{F}$ & $\mathrm{BP}$ & $\mathrm{V}, 0$ & 1200 & 1620 \\
\hline \multicolumn{8}{|l|}{ Family FORMICARIIDAE } \\
\hline Formicarius colma & Rufous-capped Antthrush & Gallito-Hormiguero de Gorro Rufo & $\mathrm{F}$ & $\mathrm{BP}$ & $V, 0$ & 1400 & 1620 \\
\hline Formicarius analis & Black-faced Antthrush & Gallito-Hormiguero de Cara Negra & $\mathrm{F}$ & $\mathrm{BP}$ & $V, 0$ & 1300 & 1500 \\
\hline Chamaeza campanisona & Short-tailed Antthrush & Rasconzuelo de Cola Corta & $\mathrm{F}$ & $\mathrm{BP}$ & $\mathrm{V}, 0$ & 1400 & 1620 \\
\hline \multicolumn{8}{|l|}{ Family FURNARIIDAE } \\
\hline Sclerurus mexicanus & Tawny-throated Leaftosser & Tira-Hoja de Garganta Anteada & $\mathrm{F}$ & $\mathrm{BP}$ & $\mathrm{V}, 0$ & 1000 & 1400 \\
\hline Sclerurus albigularis & Gray-throated Leaftosser & Tira-Hoja de Garganta Gris & $\mathrm{U}$ & $\mathrm{BP}$ & $\mathrm{V}, 0$ & 1200 & 1400 \\
\hline Furnarius leucopus & Pale-legged Hornero & Hornero de Pata Pálida & $\mathrm{F}$ & BS & $\mathrm{V}, 0$ & 900 & 1400 \\
\hline Synallaxis albigularis & Dark-breasted Spinetail & Cola-Espina de Pecho Oscuro & $F$ & $\mathrm{PC}, \mathrm{AD}$ & $V, 0$ & 1000 & 1400 \\
\hline Synallaxis hypospodia & Cinereous-breasted Spinetail & Cola-Espina de Pecho Cinéreo & $\mathrm{F}$ & $\mathrm{BL}, \mathrm{AD}$ & $\mathrm{V}, 0$ & 1000 & 1400 \\
\hline Synallaxis cherriei & Chestnut-throated Spinetail & Cola-Espina de Garganta Castaña & $\mathrm{F}$ & $\mathrm{BP}, \mathrm{BS}$ & $\mathrm{V}, 0, \mathrm{Gr}$ & 1000 & 1500 \\
\hline Synallaxis moesta & Dusky Spinetail & Cola-Espina Oscuro & $\mathrm{F}$ & BS & V & 1400 & 1500 \\
\hline Cranioleuca curtata & Ash-browed Spinetail & Cola-Espina de Ceja Ceniza & $\mathrm{U}$ & $\mathrm{BP}$ & $\mathrm{V}, 0$ & 1300 & 1620 \\
\hline Phacellodomus rufifrons & Rufous-fronted Thornbird & Espinero de Frente Rufa & C & $A D$ & $V_{1} 0$ & 900 & 1400 \\
\hline Premnoplex brunnescens & Spotted Barbtail & Cola-Púa Moteada & U & $\mathrm{BP}$ & $\mathrm{V}$ & 1300 & 1500 \\
\hline
\end{tabular}




\begin{tabular}{|c|c|c|c|c|c|c|c|}
\hline \multicolumn{3}{|c|}{ AVES DE LA SUBCUENCA DEL MISQUIYAQUILLO, MOYOBAMBA, SAN MARTÍN } & \multirow{2}{*}{ 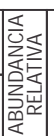 } & \multirow{2}{*}{$\begin{array}{l}\text { PREFERENCIA } \\
\text { DE HABITAT }\end{array}$} & \multirow{2}{*}{ 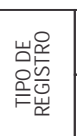 } & \multicolumn{2}{|c|}{$\begin{array}{c}\text { RANGO } \\
\begin{array}{c}\text { ALTiTUDINAL } \\
(\mathrm{m} . \mathrm{s}, \mathrm{n}, \mathrm{m})\end{array}\end{array}$} \\
\hline NOMBRE CIENTÍFICO & NOMBRE EN INGLES & NOMBRE EN ESPAÑOL & & & & \begin{tabular}{|l|} 
Rango \\
Inicial
\end{tabular} & $\begin{array}{c}\text { Rango } \\
\text { Final }\end{array}$ \\
\hline Philydor erythrocercum & Rufous-rumped Foliage-gleaner & Limpia-Follaje de Lomo Rufo & $\mathrm{U}$ & $\mathrm{BP}$ & $\mathrm{V}$ & 1400 & 1620 \\
\hline Anabazenops dorsalis & Dusky-cheeked Foliage-gleaner & Hoja-Rasquero de Mejilla Oscura & U & BS & $\mathrm{V}, 0$ & 1300 & 1350 \\
\hline Automolus ochrolaemus & Buff-throated Foliage-gleaner & Hoja-Rasquero de Garganta Anteada & $\mathrm{F}$ & $\mathrm{BP}, \mathrm{BS}$ & $\mathrm{V}, 0$ & 1000 & 1300 \\
\hline Xenops minutus & Plain Xenops & Pico-Lezna Simple & $\mathrm{F}$ & $\mathrm{BP}, \mathrm{BS}$ & $\mathrm{V}, 0$ & 1000 & 1400 \\
\hline Xenops rutilans & Streaked Xenops & Pico-Lezna Rayado & $\mathrm{F}$ & $\mathrm{BP}, \mathrm{BS}, \mathrm{PC}$ & $\mathrm{V}, 0$ & 1000 & 1620 \\
\hline \multicolumn{8}{|l|}{ Family DENDROCOLAPTIDAE } \\
\hline Dendrocincla fuliginosa & Plain-brown Woodcreeper & Trepador Pardo & $\mathrm{U}$ & $\mathrm{BP}$ & $\mathrm{V}$ & 1000 & 1300 \\
\hline Sittasomus griseicapillus & Olivaceous Woodcreeper & Trepador Oliváceo & $\mathrm{F}$ & $\mathrm{BP}, \mathrm{BS}, \mathrm{PC}$ & $V, 0$ & 1000 & 1620 \\
\hline Glyphorynchus spirurus & Wedge-billed Woodcreeper & Trepador Pico de Cuña & $\mathrm{F}$ & $\mathrm{BP}, \mathrm{BS}, \mathrm{PC}$ & $\mathrm{V}, 0$ & 900 & 1200 \\
\hline Dendrexetastes rufigula & Cinnamon-throated Woodcreeper & Trepador de Garganta Canela & $\mathrm{U}$ & $\mathrm{BP}$ & & 1100 & 1400 \\
\hline Xiphorhynchus ocellatus & Ocellated Woodcreeper & Trepador Ocelado & $\mathrm{U}$ & $\mathrm{BP}$ & $\mathrm{V}$ & 1300 & 1500 \\
\hline Xiphorhynchus elegans & Elegant Woodcreeper & Trepador Elegante & $\mathrm{F}$ & $\mathrm{BP}$ & $\mathrm{V}$ & 1000 & 1200 \\
\hline Lepidocolaptes lacrymiger & Montane Woodcreeper & Trepador Montano & $\mathrm{U}$ & $\mathrm{BP}$ & $\mathrm{V}$ & 1300 & 1620 \\
\hline Lepidocolaptes albolineatus & Lineated Woodcreeper & Trepador Lineado & $\mathrm{F}$ & $\mathrm{BP}, \mathrm{BS}$ & $\mathrm{V}$ & 1000 & 1200 \\
\hline Campylorhamphus trochilirostris & Red-billed Scythebill & Pico-Guadaña de Pico Rojo & $u$ & $\mathrm{BP}$ & $\mathrm{V}, 0$ & 1400 & 1620 \\
\hline \multicolumn{8}{|l|}{ Family TYRANNIDAE } \\
\hline Phyllomyias griseiceps & Sooty-headed Tyrannulet & Moscareta de Cabeza Tiznada & $\mathrm{F}$ & $\mathrm{BS}, \mathrm{PC}$ & $\mathrm{V}, 0$ & 1300 & 1450 \\
\hline Tyrannulus elatus & Yellow-crowned Tyrannulet & Moscareta de Corona Amarilla & $\mathrm{F}$ & $\mathrm{BS}, \mathrm{PC}$ & $\mathrm{V}, 0$ & 900 & 1050 \\
\hline Myiopagis gaimardii & Forest Elaenia & Fío-Fío de la Selva & $\mathrm{F}$ & $\mathrm{BP}, \mathrm{BS}, \mathrm{PC}$ & $\mathrm{V}, 0, \mathrm{C}$ & 1000 & 1100 \\
\hline Elaenia flavogaster & Yellow-bellied Elaenia & Fío-Fío de Vientre Amarillo & $\mathrm{F}$ & BS & $\mathrm{V}, 0$ & 900 & 1000 \\
\hline Elaenia parvirostris (NB) & Small-billed Elaenia & Fío-Fío de Pico Chico & $\mathrm{mc}$ & $\mathrm{BP}, \mathrm{BS}, \mathrm{BL}, \mathrm{PC}$ & $\mathrm{V}, 0$ & 1000 & 1400 \\
\hline Elaenia chiriquensis & Lesser Elaenia & Fío-Fío Menor & U & $\mathrm{BS}$ & $\mathrm{V}, 0$ & 900 & 1000 \\
\hline Ornithion inerme & White-lored Tyrannulet & Moscareta de Lores Blancos & $\mathrm{F}$ & $\mathrm{BP}, \mathrm{BS}$ & $\mathrm{V}, 0$ & 1000 & 1100 \\
\hline Phaeomyias murina & Mouse-colored Tyrannulet & Moscareta Murina & $\mathrm{F}$ & BS & $\mathrm{V}, 0$ & 900 & 1000 \\
\hline Zimmerius cinereicapilla & Red-billed Tyrannulet & Moscareta de Pico Rojo & $\mathrm{U}$ & $\mathrm{BP}, \mathrm{BS}, \mathrm{PC}$ & $\mathrm{V}$ & 1200 & 1400 \\
\hline Zimmerius villarejoi (E) & Mishana Tyrannulet & Moscareta de Mishana & $\mathrm{F}$ & $\mathrm{BS}, \mathrm{BL}$ & $\mathrm{V}, 0$ & 900 & 1450 \\
\hline Zimmerius chrysops & Golden-faced Tyrannulet & Moscareta de Cara Dorada & $\mathrm{F}$ & $\mathrm{BP}, \mathrm{BS}, \mathrm{PC}$ & $\mathrm{V}, 0$ & 1400 & 1620 \\
\hline Phylloscartes ophthalmicus & Marble-faced Bristle-Tyrant & Moscareta-Cerdosa de Cara Jaspeada & $\mathrm{F}$ & $\mathrm{BP}$ & $\mathrm{V}, 0$ & 1300 & 1620 \\
\hline Phylloscartes gualaquizae & Ecuadorian Tyrannulet & Moscareta Ecuatoriana & $\mathrm{U}$ & BS & $\mathrm{V}, 0$ & 1300 & 1400 \\
\hline Mionectes striaticollis & Streak-necked Flycatcher & Mosquerito de Cuello Listado & $\mathrm{F}$ & $\mathrm{BP}$ & $\mathrm{V}, 0$ & 1400 & 1620 \\
\hline Mionectes olivaceus & Olive-striped Flycatcher & Mosquerito Rayado de Olivo & $\mathrm{F}$ & $\mathrm{BP}, \mathrm{BS}$ & $\mathrm{V}, 0$ & 1000 & 1620 \\
\hline Mionectes oleagineus & Ochre-bellied Flycatcher & Mosquerito de Vientre Ocráceo & $\mathrm{F}$ & $\mathrm{BP}, \mathrm{BS}, \mathrm{PC}$ & $\mathrm{V}, 0$ & 900 & 1200 \\
\hline Leptopogon superciliaris & Slaty-capped Flycatcher & Mosquerito de Gorro Pizarroso & $\mathrm{F}$ & $\mathrm{BP}, \mathrm{BS}, \mathrm{PC}$ & $V, 0, C$ & 1000 & 1620 \\
\hline Myiornis albiventris & White-bellied Pygmy-Tyrant & Tirano-Pigmeo de Vientre Blanco & $\mathrm{F}$ & $\mathrm{BP}, \mathrm{BS}$ & $\mathrm{V}, 0$ & 1000 & 1200 \\
\hline Myiornis ecaudatus & Short-tailed Pygmy-Tyrant & Tirano-Pigmeo de Cola Corta & $\mathrm{U}$ & $\mathrm{BS}, \mathrm{BL}$ & $\mathrm{V}, 0$ & 1000 & 1450 \\
\hline Lophotriccus pileatus & Scale-crested Pygmy-Tyrant & Tirano-Pigmeo de Cresta Escamosa & $\mathrm{F}$ & $\mathrm{BP}, \mathrm{BS}$ & $\mathrm{V}, 0$ & 1200 & 1500 \\
\hline Hemitriccus zosterops & White-eyed Tody-Tyrant & Tirano-Todi de 0jo Blanco & $\mathrm{U}$ & $\mathrm{BL}$ & $\mathrm{V}$ & 1200 & 1450 \\
\hline Hemitriccus striaticollis & Stripe-necked Tody-Tyrant & Tirano-Todi de Cuello Rayado & u & $\mathrm{BS}, \mathrm{BL}$ & $\mathrm{V}, 0$ & 900 & 1000 \\
\hline Hemitriccus margaritaceiventer & Pearly-vented Tody-Tyrant & Tirano-Todi de Vientre Perlado & $\mathrm{u}$ & $\mathrm{BS}, \mathrm{BL}$ & $\mathrm{v}$ & 900 & 1300 \\
\hline Hemitriccus rufigularis & Buff-throated Tody-Tyrant & Tirano-Todi de Garganta Anteada & $\mathrm{F}$ & $\mathrm{BP}$ & $\mathrm{V}, 0, \mathrm{~F}$ & 1400 & 1620 \\
\hline Poecilotriccus capitalis & Black-and-white Tody-Flycatcher & Espatulilla Negra y Blanca & $\mathrm{F}$ & $\mathrm{BP}, \mathrm{BS}$ & $\mathrm{v}, 0$ & 1000 & 1350 \\
\hline Poecilotriccus latirostris & Rusty-fronted Tody-Flycatcher & Espatulilla de Frente Rojiza & $\mathrm{U}$ & BS & $\mathrm{V}, 0$ & 900 & 1400 \\
\hline Todirostrum cinereum & Common Tody-Flycatcher & Espatulilla Común & $\mathrm{F}$ & $\mathrm{BS}, \mathrm{PC}$ & $\mathrm{V}, 0$ & 900 & 1500 \\
\hline Todirostrum chrysocrotaphum & Yellow-browed Tody-Flycatcher & Espatulilla de Ceja Amarilla & $\mathrm{U}$ & $\mathrm{BS}, \mathrm{PC}$ & $\mathrm{V}, 0$ & 1000 & 1100 \\
\hline Rhynchocyclus olivaceus & Olivaceous Flatbill & Pico-Plano Oliváceo & $\mathrm{U}$ & $\mathrm{BP}$ & $\mathrm{V}, 0$ & 1000 & 1200 \\
\hline Tolmomyias assimilis & Yellow-margined Flycatcher & Pico-Ancho de Ala Amarilla & $\mathrm{F}$ & $\mathrm{BP}, \mathrm{BS}$ & $\mathrm{V}, 0$ & 1000 & 1300 \\
\hline Tolmomyias flaviventris & Yellow-breasted Flycatcher & Pico-Ancho de Pecho Amarillo & $\mathrm{F}$ & $\mathrm{Bp}, \mathrm{BS}, \mathrm{PC}$ & $\mathrm{V}, \mathrm{O}, \mathrm{C}$ & 1000 & 1400 \\
\hline Platyrinchus mystaceus & White-throated Spadebill & Pico-Chato de Garganta Blanca & $\mathrm{U}$ & $\mathrm{BP}$ & $\mathrm{v}, 0$ & 1200 & 1400 \\
\hline Myiophobus roraimae & Roraiman Flycatcher & Mosquerito de Roraima & $\mathrm{F}$ & $\mathrm{BP}$ & $\mathrm{V}$ & 1400 & 1620 \\
\hline Myiophobus cryptoxanthus & Olive-chested Flycatcher & Mosquerito de Pecho Olivo & $\mathrm{U}$ & BS & $\mathrm{V}, 0$ & 1200 & 1500 \\
\hline Myiobius villosus & Tawny-breasted Flycatcher & Mosquerito de Pecho Leonado & $\mathrm{U}$ & $\mathrm{BP}$ & $\mathrm{V}$ & 1200 & 1400 \\
\hline Lathrotriccus euleri & Euler's Flycatcher & Mosquerito de Euler & $\mathrm{mF}$ & $\mathrm{BP}$ & $\mathrm{V}, 0$ & 1000 & 1500 \\
\hline Empidonax alnorum (NB) & Alder Flycatcher & Mosquerito de Alisos & $\mathrm{mR}$ & $\mathrm{BS}$ & $\mathrm{V}, 0$ & 900 & 1000 \\
\hline Contopus cooperi (NB) & Olive-sided Flycatcher & Pibí Boreal & $\mathrm{mF}$ & $B S, B L, P C$ & $V, 0, C$ & 1000 & 1620 \\
\hline Contopus sordidulus (NB) & Western Wood-Pewee & Pibí Occidental & $\mathrm{mU}$ & $\mathrm{BS}, \mathrm{PC}$ & $\mathrm{V}$ & 1000 & 1620 \\
\hline Contopus virens (NB) & Eastern Wood-Pewee & Pibí Oriental & $\mathrm{mF}$ & $\mathrm{BS}, \mathrm{PC}$ & $\mathrm{V}, 0$ & 1000 & 1400 \\
\hline Knipolegus poecilurus & Rufous-tailed Tyrant & Viudita de Cola Rufa & $\mathrm{U}$ & $\mathrm{BL}$ & $\mathrm{V}$ & 1200 & 1400 \\
\hline Muscisaxicola fluviatilis & Little Ground-Tyrant & Dormilona Enana & $R$ & $A D$ & $\mathrm{~V}$ & 900 & 1000 \\
\hline Colonia colonus & Long-tailed Tyrant & Tirano de Cola Larga & $\mathrm{F}$ & $\mathrm{BS}, \mathrm{PC}$ & $\mathrm{V}, 0$ & 1000 & 1620 \\
\hline Legatus leucophaius & Piratic Flycatcher & Mosquero Pirata & $\mathrm{F}$ & $\mathrm{BS}, \mathrm{PC}$ & $\mathrm{V}, 0$ & 900 & 1200 \\
\hline Myiozetetes similis & Social Flycatcher & Mosquero Social & $\mathrm{F}$ & $\mathrm{BS}, \mathrm{PC}$ & $\mathrm{V}, 0$ & 900 & 1400 \\
\hline Myiozetetes granadensis & Gray-capped Flycatcher & Mosquero de Gorro Gris & $\mathrm{F}$ & $\mathrm{BS}, \mathrm{PC}$ & $\mathrm{V}, 0$ & 1000 & 1200 \\
\hline Pitangus sulphuratus & Great Kiskadee & Bienteveo Grande & $\mathrm{F}$ & $\mathrm{BS}, \mathrm{PC}$ & $\mathrm{V}, 0$ & 900 & 1200 \\
\hline
\end{tabular}




\begin{tabular}{|c|c|c|c|c|c|c|c|}
\hline \multicolumn{3}{|c|}{ AVES DE LA SUBCUENCA DEL MISQUIYAQUILLO, MOYOBAMBA, SAN MARTÍN } & \multirow{2}{*}{$\begin{array}{l}5 \\
0 \\
05 \\
5 \\
3 \\
3 \\
0 \\
0\end{array}$} & \multirow{2}{*}{$\begin{array}{l}\text { PREFERENCIA } \\
\text { DE HABITAT }\end{array}$} & \multirow{2}{*}{ 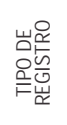 } & \multicolumn{2}{|c|}{$\begin{array}{c}\text { RANGO } \\
\text { ALTITUDINAL } \\
(\mathrm{m} . \mathrm{s}, \mathrm{n}, \mathrm{m})\end{array}$} \\
\hline NOM BRE CIENTÍFICO & NOMBRE EN INGLES & NOMBRE EN ESPAÑOL & & & & \begin{tabular}{l|l} 
Rango \\
Inicial
\end{tabular} & $\begin{array}{c}\text { Rango } \\
\text { Final }\end{array}$ \\
\hline Conopias cinchoneti & Lemon-browed Flycatcher & Mosquero de Ceja Limón & $\mathrm{U}$ & $\mathrm{BP}$ & $\mathrm{V}$ & 1200 & 1400 \\
\hline Myiodynastes chrysocephalus & Golden-crowned Flycatcher & Mosquero de Corona Dorada & $\mathrm{R}$ & $\mathrm{BP}$ & $\mathrm{V}, 0$ & 1500 & 1620 \\
\hline Myiodynastes maculatus & Streaked Flycatcher & Mosquero Rayado & $\mathrm{mF}$ & $\mathrm{BS}, \mathrm{PC}$ & $\mathrm{V}, 0$ & 900 & 1400 \\
\hline Megarynchus pitangua & Boat-billed Flycatcher & Mosquero Picudo & $\mathrm{F}$ & $\mathrm{BS}, \mathrm{PC}$ & $\mathrm{V}, 0$ & 900 & 1200 \\
\hline Empidonomus aurantioatrocristatus NB & Crowned Slaty Flycatcher & Mosquero-Pizarroso Coronado & $\mathrm{mR}$ & BL & $\mathrm{V}$ & 1300 & 1400 \\
\hline Tyrannus melancholicus & Tropical Kingbird & Tirano Tropical & $\mathrm{F}$ & $\mathrm{BS}, \mathrm{PC}$ & $\mathrm{V}, 0$ & 900 & 1620 \\
\hline Rhytipterna simplex & Grayish Mourner & Plañidero Grisáceo & $\mathrm{U}$ & $\mathrm{BL}$ & V, 0 & 1100 & 1400 \\
\hline Myiarchus ferox & Short-crested Flycatcher & Copetón de Cresta Corta & $\mathrm{F}$ & $\mathrm{BS}, \mathrm{PC}$ & $\mathrm{V}, 0$ & 900 & 1100 \\
\hline \multicolumn{8}{|l|}{ Family COTINGIDAE } \\
\hline Pipreola frontalis & Scarlet-breasted Fruiteater & Frutero de Pecho Escarlata & $\mathrm{F}$ & $\mathrm{BP}$ & $\mathrm{V}, 0$ & 1500 & 1620 \\
\hline Pipreola chlorolepidota & Fiery-throated Fruiteater & Frutero Garganta de Fuego & $\mathrm{F}$ & $\mathrm{BP}, \mathrm{BS}$ & $\mathrm{V}, 0$ & 1000 & 1500 \\
\hline Ampelioides tschudii & Scaled Fruiteater & Frutero Escamoso & $\mathrm{U}$ & $\mathrm{BP}$ & $\mathrm{V}, 0$ & 1400 & 1620 \\
\hline Rupicola peruvianus & Andean Cock-of-the-rock & Gallito-de-las-Rocas Andino & $R$ & $\mathrm{BP}$ & $V, R$ & 1200 & 1400 \\
\hline Snowornis subalaris & Gray-tailed Piha & Piha de Cola Gris & $\mathrm{U}$ & $\mathrm{BP}$ & $\mathrm{v}, 0$ & 1350 & 1500 \\
\hline Snowornis cryptolophus & Olivaceous Piha & Piha Olivácea & $\mathrm{F}$ & $\mathrm{BP}$ & $\mathrm{v}, 0$ & 1350 & 1500 \\
\hline \multicolumn{8}{|l|}{ Family PIPRIDAE } \\
\hline Machaeropterus regulus & Striped Manakin & Saltarín Rayado & $\mathrm{F}$ & $\mathrm{BP}, \mathrm{BL}$ & $\mathrm{v}, 0$ & 1100 & 1350 \\
\hline Machaeropterus pyrocephalus & Fiery-capped Manakin & Saltarín Gorro de Fuego & $\mathrm{F}$ & BS & $\mathrm{V}, \mathrm{O}, \mathrm{C}$ & 1000 & 1300 \\
\hline Lepidothrix isidorei & Blue-rumped Manakin & Saltarín de Lomo Azul & $\mathrm{U}$ & $\mathrm{BP}$ & $\mathrm{V}, 0$ & 1300 & 1620 \\
\hline Xenopipo holochlora & Green Manakin & Saltarín Verde & $\mathrm{U}$ & $\mathrm{BP}$ & $\mathrm{V}$ & 1100 & 1200 \\
\hline Pipra pipra & White-crowned Manakin & Saltarín de Corona Blanca & $\mathrm{U}$ & $\mathrm{BP}$ & $\mathrm{V}$ & 1500 & 1620 \\
\hline Pipra erythrocephala & Golden-headed Manakin & Saltarín de Cabeza Dorada & $\mathrm{F}$ & $\mathrm{BP}, \mathrm{BS}, \mathrm{PC}$ & $\mathrm{V}, 0$ & 1000 & 1300 \\
\hline \multicolumn{8}{|l|}{ Family TITYRIDAE } \\
\hline Tityra semifasciata & Masked Tityra & Titira Enmascarada & $\mathrm{F}$ & $\mathrm{BS}, \mathrm{PC}$ & $\mathrm{V}, 0$ & 1000 & 1600 \\
\hline Schiffornis turdina & Thrush-like Schiffornis & Shifornis Pardo & $\mathrm{F}$ & $\mathrm{BP}$ & $\mathrm{v}, 0$ & 1500 & 1620 \\
\hline Laniisoma elegans & Elegant Mourner & Plañidero Elegante & $\mathrm{R}$ & $\mathrm{BP}$ & $\mathrm{V}, 0$ & 1300 & 1500 \\
\hline Pachyramphus viridis & Green-backed Becard & Cabezón de Dorso Verde & $\mathrm{U}$ & $\mathrm{BP}, \mathrm{BS}$ & $\mathrm{V}$ & 1300 & 1500 \\
\hline Pachyramphus castaneus & Chestnut-crowned Becard & Cabezón de Corona Castaña & $\mathrm{U}$ & $\mathrm{PC}$ & $\mathrm{V}, 0$ & 1300 & 1400 \\
\hline Pachyramphus polychopterus & White-winged Becard & Cabezón de Ala Blanca & $\mathrm{F}$ & $\mathrm{BS}, \mathrm{PC}$ & $\mathrm{V}, 0$ & 1000 & 1400 \\
\hline Pachyramphus albogriseus & Black-and-white Becard & Cabezón Blanco y Negro & $\mathrm{F}$ & $\mathrm{BP}$ & $\mathrm{V}, 0$ & 1500 & 1620 \\
\hline \multicolumn{8}{|l|}{ GENERA INCERTAE SEDIS } \\
\hline Piprites chloris & Wing-barred Piprites & Piprites de Ala Barrada & $\mathrm{F}$ & $B P, B S, P C$ & $\mathrm{~V}, 0$ & 1000 & 1500 \\
\hline \multicolumn{8}{|l|}{ Family VIREONIDAE } \\
\hline Vireolanius leucotis & Slaty-capped Shrike-Vireo & Vireón de Gorro Apizarrado & $\mathrm{F}$ & $\mathrm{BP}$ & $\mathrm{V}, 0$ & 1100 & 1400 \\
\hline Vireo olivaceus & Red-eyed Vireo & Víreo de Ojo Rojo & $\mathrm{F}$ & $B P, B S, P C$ & $\mathrm{~V}, 0$ & 900 & 1620 \\
\hline Hylophilus thoracicus & Lemon-chested Greenlet & Verdillo de Pecho Limón & $\mathrm{U}$ & $\mathrm{BP}, \mathrm{BS}$ & $\mathrm{V}, 0$ & 1000 & 1200 \\
\hline Hylophilus olivaceus & Olivaceous Greenlet & Verdillo Oliváceo & $\mathrm{F}$ & $\mathrm{BP}, \mathrm{BS}, \mathrm{PC}$ & $\mathrm{V}, 0$ & 1000 & 1500 \\
\hline Hylophilus ochraceiceps & Tawny-crowned Greenlet & Verdillo de Corona Leonada & $\mathrm{F}$ & $\mathrm{BP}$ & $\mathrm{V}, 0$ & 1100 & 1200 \\
\hline \multicolumn{8}{|l|}{ Family CORVIDAE } \\
\hline Cyanocorax yncas & Green Jay & Urraca Verde & $\mathrm{C}$ & $\mathrm{BP}$ & $\mathrm{V}, 0$ & 1300 & 1620 \\
\hline \multicolumn{8}{|l|}{ Family HIRUNDINIDAE } \\
\hline Pygochelidon cyanoleuca & Blue-and-white Swallow & Golondrina Azul y Blanca & $\mathrm{C}$ & $B S, A D$ & $\mathrm{~V}, 0$ & 900 & 1620 \\
\hline Stelgidopteryx ruficollis & Southern Rough-winged Swallow & Golondrina Ala-Rasposa Sureña & $\mathrm{F}$ & BS & V & 1000 & 1200 \\
\hline Progne chalybea & Gray-breasted Martin & Martín de Pecho Gris & $\mathrm{U}$ & $A D$ & V & 900 & 1000 \\
\hline \multicolumn{8}{|l|}{ Family TROGLODYTIDAE } \\
\hline Microcerculus marginatus & Scaly-breasted Wren & Cucarachero de Pecho Escamoso & $\mathrm{F}$ & $\mathrm{BP}$ & $\mathrm{V}, 0$ & 1000 & 1450 \\
\hline Odontorchilus branickii & Gray-mantled Wren & Cucarachero de Dorso Gris & $\mathrm{F}$ & $\mathrm{BP}$ & $\mathrm{V}$ & 1500 & 1620 \\
\hline Troglodytes aedon & House Wren & Cucarachero Común & $\mathrm{F}$ & $\mathrm{PC}, \mathrm{AD}$ & $\mathrm{v}, 0$ & 900 & 1620 \\
\hline Campylorhynchus turdinus & Thrush-like Wren & Cucarachero Zorzal & $\mathrm{F}$ & $\mathrm{BS}, \mathrm{PC}$ & $\mathrm{v}, 0$ & 1400 & 1500 \\
\hline Pheugopedius coraya & Coraya Wren & Cucarachero Coraya & $\mathrm{F}$ & $\mathrm{BP}, \mathrm{BS}$ & $\mathrm{V}, 0$ & 1000 & 1400 \\
\hline Henicorhina leucophrys & Gray-breasted Wood-Wren & Cucarachero-Montés de Pecho Gris & $\mathrm{F}$ & $\mathrm{BP}$ & $\mathrm{V}, 0$ & 1500 & 1620 \\
\hline Family TURDIDAE & & & & & & & \\
\hline Catharus ustulatus (NB) & Swainson's Thrush & Zorzal de Swainson & $\mathrm{mc}$ & $\mathrm{BP}, \mathrm{BS}, \mathrm{BL}, \mathrm{PC}, \mathrm{AD}$ & $\mathrm{V}, 0$ & 900 & 1620 \\
\hline Turdus hauxwelli & Hauxwell's Thrush & Zorzal de Hauxwell & $\mathrm{U}$ & $\mathrm{BS}, \mathrm{PC}$ & $\mathrm{V}, 0$ & 900 & 1200 \\
\hline Turdus ignobilis & Black-billed Thrush & Zorzal de Pico Negro & $\mathrm{C}$ & $\mathrm{BS}, \mathrm{PC}$ & $\mathrm{V}, 0$ & 900 & 1200 \\
\hline Turdus albicollis & White-necked Thrush & Zorzal de Cuello Blanco & $\mathrm{F}$ & $\mathrm{BP}$ & $\mathrm{V}, 0$ & 1000 & 1350 \\
\hline Family THRAUPIDAE & & & & & & & \\
\hline Schistochlamys melanopis & Black-faced Tanager & Tangara de Cara Negra & $\mathrm{F}$ & $\mathrm{BS}, \mathrm{BL}$ & $\mathrm{V}, 0$ & 900 & 1450 \\
\hline Cissopis leverianus & Magpie Tanager & Tangara Urraca & $\mathrm{F}$ & $\mathrm{BS}, \mathrm{PC}$ & $\mathrm{V}, 0$ & 1000 & 1600 \\
\hline Trichothraupis melanops & Black-goggled Tanager & Tangara de Anteojos & $\mathrm{F}$ & $\mathrm{BP}$ & $V, 0, C$ & 1100 & 1400 \\
\hline Tachyphonus rufiventer & Yellow-crested Tanager & Tangara de Cresta Amarilla & $\mathrm{F}$ & $\mathrm{BP}, \mathrm{BS}$ & $\mathrm{v}, 0$ & 1000 & 1250 \\
\hline Tachyphonus rufus & White-lined Tanager & Tangara de Líneas Blancas & $\mathrm{F}$ & $\mathrm{BS}, \mathrm{PC}$ & $\mathrm{V}, 0$ & 1000 & 1500 \\
\hline Tachyphonus phoenicius & Red-shouldered Tanager & Tangara de Hombro Rojo & $\mathrm{F}$ & $\mathrm{BL}$ & $\mathrm{V}, 0$ & 1200 & 1450 \\
\hline
\end{tabular}




\begin{tabular}{|c|c|c|c|c|c|c|c|}
\hline \multicolumn{3}{|c|}{ AVES DE LA SUBCUENCA DEL MISQUIYAQUILLO, MOYOBAMBA, SAN MARTÍN } & \multirow{2}{*}{ 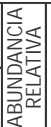 } & \multirow{2}{*}{$\begin{array}{c}\text { PREFERENCIA } \\
\text { DE HABITAT }\end{array}$} & \multirow{2}{*}{$\begin{array}{l}\text { 宸 } \\
8 \\
\text { F要 }\end{array}$} & \multicolumn{2}{|c|}{$\begin{array}{c}\text { RANGO } \\
\begin{array}{c}\text { ALTITUDINAL } \\
(\mathrm{m}, \mathrm{s}, \mathrm{n}, \mathrm{m})\end{array}\end{array}$} \\
\hline NOMBRE CIENTÍFICO & NOMBRE EN INGLES & NOMBRE EN ESPAÑOL & & & & \begin{tabular}{|l|} 
Rango \\
Inicial
\end{tabular} & $\begin{array}{l}\text { Rango } \\
\text { Final }\end{array}$ \\
\hline Lanio fulvus & Fulvous Shrike-Tanager & Tangara Leonada & $\mathrm{U}$ & $\mathrm{BP}$ & $\mathrm{V}, 0$ & 1400 & 1500 \\
\hline Ramphocelus melanogaster (E) & Black-bellied Tanager & Tangara de Vientre Negro & $\mathrm{F}$ & $\mathrm{BS}, \mathrm{PC}$ & $\mathrm{V}, 0, \mathrm{~F}$ & 900 & 1620 \\
\hline Thraupis episcopus & Blue-gray Tanager & Tangara Azuleja & $\mathrm{F}$ & $\mathrm{BS}, \mathrm{PC}$ & $\mathrm{V}, 0$ & 900 & 1620 \\
\hline Thraupis palmarum & Palm Tanager & Tangara de Palmeras & $\mathrm{F}$ & $\mathrm{BS}, \mathrm{PC}$ & $\mathrm{V}, 0$ & 900 & 1600 \\
\hline Anisognathus somptuosus & Blue-winged Mountain-Tanager & Tangara-de-Montaña de Ala Azul & $\mathrm{U}$ & $\mathrm{BP}$ & $\mathrm{V}$ & 1500 & 1620 \\
\hline Chlorochrysa calliparaea & Orange-eared Tanager & Tangara de Oreja Naranja & $\mathrm{F}$ & $\mathrm{BP}$ & $\mathrm{v}$ & 1500 & 1620 \\
\hline Tangara ruficervix & Golden-naped Tanager & Tangara de Nuca Dorada & $\mathrm{F}$ & $\mathrm{BP}$ & $\mathrm{V}, 0$ & 1500 & 1620 \\
\hline Tangara cayana & Burnished-buff Tanager & Tangara de Anteado Bruñido & $\mathrm{U}$ & BS & $\mathrm{V}, 0$ & 900 & 1000 \\
\hline Tangara nigrocincta & Masked Tanager & Tangara Enmascarada & $\mathrm{F}$ & $\mathrm{BP}, \mathrm{BS}, \mathrm{BL}$ & $\mathrm{V}, 0$ & 1100 & 1300 \\
\hline Tangara cyanicollis & Blue-necked Tanager & Tangara de Cuello Azul & $\mathrm{F}$ & $\mathrm{BP}, \mathrm{BS}, \mathrm{BL}, \mathrm{PC}$ & $\mathrm{V}, 0$ & 900 & 1620 \\
\hline Tangara xanthogastra & Yellow-bellied Tanager & Tangara de Vientre Amarillo & $\mathrm{F}$ & $\mathrm{BP}, \mathrm{BS}, \mathrm{PC}$ & $\mathrm{V}, 0$ & 1000 & 1500 \\
\hline Tangara punctata & Spotted Tanager & Tangara Moteada & $\mathrm{F}$ & $\mathrm{BP}, \mathrm{BS}, \mathrm{BL}$ & $\mathrm{V}, 0$ & 1100 & 1620 \\
\hline Tangara mexicana & Turquoise Tanager & Tangara Turquesa & $u$ & $\mathrm{BS}, \mathrm{PC}$ & $\mathrm{V}, 0$ & 900 & 1000 \\
\hline Tangara chilensis & Paradise Tanager & Tangara del Paraíso & $\mathrm{C}$ & $\mathrm{BP}, \mathrm{BS}, \mathrm{BL}, \mathrm{PC}$ & $\mathrm{V}, 0$ & 900 & 1500 \\
\hline Tangara gyrola & Bay-headed Tanager & Tangara de Cabeza Baya & $\mathrm{F}$ & $\mathrm{BP}, \mathrm{BS}, \mathrm{PC}$ & $\mathrm{V}, 0$ & 1000 & 1620 \\
\hline Tangara chrysotis & Golden-eared Tanager & Tangara de Oreja Dorada & $\mathrm{F}$ & $\mathrm{BP}$ & $\mathrm{V}, 0$ & 1300 & 1620 \\
\hline Tangara schrankii & Green-and-gold Tanager & Tangara Verde y Dorada & $\mathrm{F}$ & $\mathrm{BP}, \mathrm{BS}, \mathrm{PC}$ & $\mathrm{V}, 0$ & 1000 & 1600 \\
\hline Tangara arthus & Golden Tanager & Tangara Dorada & $\mathrm{U}$ & $\mathrm{BP}$ & $\mathrm{V}, 0$ & 1300 & 1620 \\
\hline Tersina viridis & Swallow Tanager & Azulejo Golondrina & $\mathrm{F}$ & $\mathrm{BS}, \mathrm{BL}$ & $\mathrm{V}, 0$ & 900 & 1450 \\
\hline Dacnis lineata & Black-faced Dacnis & Dacnis de Cara Negra & $\mathrm{F}$ & $\mathrm{BP}, \mathrm{BS}, \mathrm{BL}, \mathrm{PC}$ & $\mathrm{V}, 0$ & 900 & 1300 \\
\hline Dacnis cayana & Blue Dacnis & Dacnis Azul & $\mathrm{F}$ & $\mathrm{BP}, \mathrm{BS}, \mathrm{BL}, \mathrm{PC}$ & $\mathrm{V}, 0$ & 900 & 1500 \\
\hline Cyanerpes caeruleus & Purple Honeycreeper & Mielero Púrpura & $\mathrm{F}$ & $\mathrm{BP}, \mathrm{BS}, \mathrm{BL}, \mathrm{PC}$ & $\mathrm{V}, 0$ & 900 & 1400 \\
\hline Chlorophanes spiza & Green Honeycreeper & Mielero Verde & $\mathrm{F}$ & $\mathrm{BP}, \mathrm{BS}, \mathrm{BL}, \mathrm{PC}$ & $\mathrm{V}, 0$ & 900 & 1400 \\
\hline Iridophanes pulcherrimus & Golden-collared Honeycreeper & Mielero de Collar Dorado & $\mathrm{U}$ & $\mathrm{BP}$ & V & 1500 & 1620 \\
\hline Hemithraupis guira & Guira Tanager & Tangara Guira & $\mathrm{U}$ & $\mathrm{BP}, \mathrm{BS}, \mathrm{PC}$ & $\mathrm{V}, 0$ & 1000 & 1500 \\
\hline Hemithraupis flavicollis & Yellow-backed Tanager & Tangara de Dorso Amarillo & $\mathrm{U}$ & $\mathrm{BP}$ & $\mathrm{V}, 0$ & 1100 & 1300 \\
\hline \multicolumn{8}{|l|}{ INCERTAE SEDIS } \\
\hline Coereba flaveola & Bananaquit & Mielero Común & $\mathrm{F}$ & $\mathrm{BP}, \mathrm{BS}, \mathrm{BL}, \mathrm{PC}$ & $V, 0, C$ & 900 & 1620 \\
\hline Tiaris obscurus & Dull-colored Grassquit & Semillero Pardo & $\mathrm{F}$ & $B S, P C, A D$ & $\mathrm{v}, 0$ & 900 & 1400 \\
\hline Saltator grossus & Slate-colored Grosbeak & Picogrueso de Pico Rojo & $\mathrm{F}$ & $\mathrm{BP}$ & $\mathrm{V}, 0$ & 1000 & 1300 \\
\hline Saltator maximus & Buff-throated Saltator & Saltador de Garganta Anteada & $\mathrm{F}$ & $\mathrm{BP}, \mathrm{BS}, \mathrm{BL}, \mathrm{PC}$ & $\mathrm{V}, 0$ & 1000 & 1620 \\
\hline Saltator coerulescens & Grayish Saltator & Saltador Grisáceo & $\mathrm{F}$ & $\mathrm{BS}, \mathrm{PC}$ & $\mathrm{V}, 0$ & 900 & 1400 \\
\hline \multicolumn{8}{|l|}{ Family EMBERIZIDAE } \\
\hline Zonotrichia capensis & Rufous-collared Sparrow & Gorrión de Collar Rufo & $\mathrm{F}$ & $A D$ & $\mathrm{~V}, 0$ & 900 & 1620 \\
\hline Ammodramus aurifrons & Yellow-browed Sparrow & Gorrión de Ceja Amarilla & $\mathrm{F}$ & $A D$ & $\mathrm{~V}, 0$ & 900 & 1400 \\
\hline Emberizoides herbicola & Wedge-tailed Grass-Finch & Sabanero Cola de Cuña & $\mathrm{F}$ & $A D$ & $\mathrm{~V}, 0$ & 900 & 1000 \\
\hline Volatinia jacarina & Blue-black Grassquit & Semillerito Negro Azulado & $\mathrm{F}$ & $A D$ & $\mathrm{~V}, 0$ & 900 & 1400 \\
\hline Sporophila luctuosa & Black-and-white Seedeater & Espiguero Negro y Blanco & $\mathrm{u}$ & $A D$ & $\mathrm{~V}, 0$ & 1000 & 1200 \\
\hline Sporophila castaneiventris & Chestnut-bellied Seedeater & Espiguero de Vientre Castaño & $\mathrm{F}$ & $A D$ & $\mathrm{~V}, 0$ & 900 & 1200 \\
\hline Oryzoborus angolensis & Chestnut-bellied Seed-Finch & Semillero de Vientre Castaño & $\mathrm{U}$ & $\mathrm{BS}, \mathrm{AD}$ & $\mathrm{V}, 0$ & 900 & 1300 \\
\hline Oryzoborus atrirostris & Black-billed Seed-Finch & Semillero de Pico Negro & $\mathrm{U}$ & $A D$ & $\mathrm{~V}, 0$ & 900 & 1000 \\
\hline Arremon aurantiirostris & Orange-billed Sparrow & Gorrión de Pico Naranja & $\mathrm{F}$ & $\mathrm{BP}, \mathrm{BS}$ & $\mathrm{V}, 0$ & 1000 & 1200 \\
\hline Arremon brunneinucha & Chestnut-capped Brush-Finch & Matorralero de Gorro Castaño & $\mathrm{F}$ & $\mathrm{BP}$ & $\mathrm{V}, 0$ & 1200 & 1620 \\
\hline Chlorospingus flavigularis & Yellow-throated Bush-Tanager & Tangara-Montesa de Garganta Amarilla & $\mathrm{F}$ & $\mathrm{BP}$ & $\mathrm{V}, 0$ & 1400 & 1620 \\
\hline \multicolumn{8}{|l|}{ Family CARDINALIDAE } \\
\hline Piranga flava & Hepatic Tanager & Piranga Bermeja & $\mathrm{F}$ & $\mathrm{BL}$ & $\mathrm{V}, 0$ & 1200 & 1450 \\
\hline Piranga rubra (NB) & Summer Tanager & Piranga Roja & $\mathrm{mF}$ & $\mathrm{BP}, \mathrm{BS}, \mathrm{PC}$ & $\mathrm{V}, 0$ & 900 & 1500 \\
\hline Piranga olivacea (NB) & Scarlet Tanager & Piranga Escarlata & $\mathrm{mF}$ & $\mathrm{BP}, \mathrm{BS}, \mathrm{PC}$ & $\mathrm{V}, 0$ & 900 & 1500 \\
\hline Piranga leucoptera & White-winged Tanager & Piranga de Ala Blanca & $\mathrm{U}$ & $\mathrm{BP}$ & $\mathrm{V}, 0$ & 1500 & 1620 \\
\hline Habia rubica & Red-crowned Ant-Tanager & Tangara-Hormiguera de Corona Roja & $\mathrm{F}$ & $\mathrm{BP}, \mathrm{BS}$ & $\mathrm{V}, 0$ & 1000 & 1300 \\
\hline \multicolumn{8}{|l|}{ Family PARULIDAE } \\
\hline Parula pitiayumi & Tropical Parula & Parula Tropical & $\mathrm{F}$ & $\mathrm{BP}, \mathrm{BS}, \mathrm{BL}, \mathrm{PC}$ & $\mathrm{V}, 0$ & 1000 & 1620 \\
\hline Dendroica striata (NB) & Blackpoll Warbler & Reinita Estriada & $\mathrm{mF}$ & $\mathrm{BL}, \mathrm{PC}$ & $\mathrm{V}$ & 1000 & 1300 \\
\hline Dendroica fusca (NB) & Blackburnian Warbler & Reinita de Garganta Naranja & $\mathrm{mF}$ & $\mathrm{BP}, \mathrm{BS}, \mathrm{PC}$ & $\mathrm{V}, 0$ & 1000 & 1620 \\
\hline Dendroica cerulea (NB) & Cerulean Warbler & Reinita Cerúlea & $\mathrm{mF}$ & $\mathrm{BP}, \mathrm{BS}, \mathrm{BL}, \mathrm{PC}$ & $\mathrm{V}, 0$ & 1000 & 1500 \\
\hline Setophaga ruticilla (NB) & American Redstart & Candelita Americana & $\mathrm{mR}$ & $\mathrm{BS}, \mathrm{PC}$ & $\mathrm{V}$ & 900 & 1200 \\
\hline Wilsonia canadensis (NB) & Canada Warbler & Reinita de Canada & $\mathrm{mc}$ & $\mathrm{BP}, \mathrm{BS}, \mathrm{PC}$ & $\mathrm{V}, 0$ & 900 & 1600 \\
\hline Myioborus miniatus & Slate-throated Redstart & Candelita de Garganta Plomiza & $\mathrm{F}$ & $\mathrm{BP}$ & $\mathrm{V}, 0$ & 1200 & 1620 \\
\hline Basileuterus tristriatus & Three-striped Warbler & Reinita de Cabeza Listada & $\mathrm{U}$ & $\mathrm{BP}$ & $\mathrm{V}, 0$ & 1500 & 1620 \\
\hline Phaeothlypis fulvicauda & Buff-rumped Warbler & Reinita de Lomo Anteado & $\mathrm{F}$ & $\mathrm{BP}$ & $V, 0$ & 1000 & 1300 \\
\hline
\end{tabular}




\begin{tabular}{|c|c|c|c|c|c|c|c|}
\hline \multicolumn{3}{|c|}{ AVES DE LA SUBCUENCA DEL MISQUIYAQUILLO, MOYOBAM BA, SAN MARTÍN } & \multirow{2}{*}{ 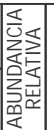 } & \multirow{2}{*}{$\begin{array}{l}\text { PREFERENCIA } \\
\text { DE HABITAT }\end{array}$} & \multirow{2}{*}{ 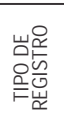 } & \multicolumn{2}{|c|}{$\begin{array}{c}\text { RANGO } \\
\text { ALTITUDINAL } \\
(\mathrm{m} . \mathrm{s} \cdot \mathrm{n} . \mathrm{m})\end{array}$} \\
\hline NOMBRE CIENTÍFICO & NOMBRE EN INGLES & NOMBRE EN ESPAÑOL & & & & $\begin{array}{l}\text { Rango } \\
\text { Inicial }\end{array}$ & $\begin{array}{l}\text { Rango } \\
\text { Final }\end{array}$ \\
\hline \multicolumn{8}{|l|}{ Family ICTERIDAE } \\
\hline Psarocolius angustifrons & Russet-backed Oropendola & Oropéndola de Dorso Bermejo & $\mathrm{F}$ & $\mathrm{BP}$ & $\mathrm{V}, 0$ & 1000 & 1300 \\
\hline Psarocolius decumanus & Crested Oropendola & Oropéndola Crestada & $\mathrm{C}$ & $\mathrm{BP}, \mathrm{BS}, \mathrm{PC}$ & $\mathrm{V}, 0$ & 900 & 1620 \\
\hline Cacicus solitarius & Solitary Black Cacique & Cacique Solitario & $\mathrm{R}$ & BS & $\mathrm{V}, 0$ & 900 & 1000 \\
\hline Cacicus cela & Yellow-rumped Cacique & Cacique de Lomo Amarillo & c & $\mathrm{BP}, \mathrm{BS}, \mathrm{PC}$ & $\mathrm{V}, 0$ & 900 & 1620 \\
\hline Cacicus haemorrhous & Red-rumped Cacique & Cacique de Lomo Rojo & $U$ & $\mathrm{BP}, \mathrm{BS}, \mathrm{PC}$ & $\mathrm{V}, 0$ & 900 & 1620 \\
\hline Icterus croconotus & Orange-backed Troupial & Turpial de Dorso Naranja & $\mathrm{F}$ & $\mathrm{BS}, \mathrm{PC}$ & $\mathrm{V}, 0$ & 900 & 1450 \\
\hline Molothrus oryzivorus & Giant Cowbird & Tordo Gigante & $\mathrm{F}$ & BS, PC & $\mathrm{V}, 0$ & 900 & 1450 \\
\hline \multicolumn{8}{|l|}{ Family FRINGILLIDAE } \\
\hline Sporagra olivacea & Olivaceous Siskin & Jilguero Oliváceo & $\mathrm{U}$ & $\mathrm{BP}$ & $\mathrm{V}, 0$ & 1300 & 1620 \\
\hline Euphonia chlorotica & Purple-throated Euphonia & Eufonia de Garganta Púrpura & $\mathrm{F}$ & $\mathrm{BS}, \mathrm{PC}$ & $\mathrm{V}, 0$ & 900 & 1400 \\
\hline Euphonia laniirostris & Thick-billed Euphonia & Eufonia de Pico Grueso & $\mathrm{F}$ & $\mathrm{BP}, \mathrm{BS}, \mathrm{PC}$ & $\mathrm{V}, 0$ & 1000 & 1200 \\
\hline Euphonia cyanocephala & Golden-rumped Euphonia & Eufonia de Lomo Dorado & U & $\mathrm{BL}$ & $\mathrm{V}, 0$ & 1300 & 1620 \\
\hline Euphonia chrysopasta & Golden-bellied Euphonia & Eufonia de Vientre Dorado & U & $\mathrm{BS}, \mathrm{PC}$ & $\mathrm{V}, 0$ & 1300 & 1620 \\
\hline Euphonia mesochrysa & Bronze-green Euphonia & Eufonia Bronce y Verde & $\mathrm{F}$ & $\mathrm{BP}, \mathrm{BS}, \mathrm{PC}$ & $\mathrm{V}, 0$ & 1300 & 1450 \\
\hline Euphonia minuta & White-vented Euphonia & Eufonia de Subcaudales Blancas & $\mathrm{F}$ & $\mathrm{BS}, \mathrm{PC}$ & $\mathrm{V}, 0$ & 900 & 1200 \\
\hline Euphonia xanthogaster & Orange-bellied Euphonia & Eufonia de Vientre Naranja & $\mathrm{F}$ & $\mathrm{BP}, \mathrm{BS}, \mathrm{BL}, \mathrm{PC}$ & $\mathrm{V}, 0$ & 1000 & 1620 \\
\hline Euphonia rufiventris & Rufous-bellied Euphonia & Eufonia de Vientre Rufo & U & $\mathrm{BL}$ & $\mathrm{v}$ & 1200 & 1400 \\
\hline Chlorophonia cyanea & Blue-naped Chlorophonia & Clorofonia de Nuca Azul & $\mathrm{F}$ & $\mathrm{BP}, \mathrm{BS}, \mathrm{BL}, \mathrm{PC}$ & $\mathrm{V}, 0$ & 1000 & 1620 \\
\hline
\end{tabular}

